\title{
Managementreformen in internationalen Organisationen: Eine vergleichende Analyse der Europäischen Kommission und des OECD-Sekretariats
}

\author{
Christoph Knill / Tim Balint
}

Im vorliegenden Artikel werden die Reformentwicklungen des Personalmanagements im OECDSekretariat und in der Europäischen Kommission vergleichend untersucht. Im Mittelpunkt steht die Frage, inwieweit beide Organisationen ihre Managementstrukturen an den Idealtypus des New Public Management (NPM) angepasst haben. Die empirischen Befunde zeigen, dass das Personalmanagement der Kommission weitreichender reformiert wurde und nun eher dem NPM entspricht als das des OECD-Sekretariats. Dieses Ergebnis ist aus zwei Gründen überraschend: Erstens erscheint es paradox, dass gerade die OECD, die auf internationaler Ebene als zentraler Verfechter von NPM gilt, bei der eigenen Umsetzung dieser Reformen hinterherhinkt. Zweitens steht das Ergebnis im Widerspruch zu theoretischen Erwartungen, wie sie sich aus dem Konzept des institutionellen Isomorphismus ergeben. Um vor diesem Hintergrund dennoch zu einer befriedigenden Erklärung der überraschenden Ergebnisse zu gelangen, ist es erforderlich, einschlägige Theorien institutioneller Isomorphie im Hinblick auf die Wirksamkeitsbedingungen ihrer kausalen Mechanismen zu ergänzen.

Schlagwörter: New Public Management, Verwaltungsreform, OECD, Europäische Kommission

\section{Einleitung*}

Es gibt mittlerweile unzählige Studien, die sich mit Ursachen und Ergebnissen nationaler Verwaltungsreformen befassen. Dies gilt insbesondere mit Blick auf die Einführung des sogenannten New Public Management (NPM), das sich seit Beginn der 1990er Jahre als dominantes Reformparadigma auf globaler Ebene etabliert hat (vgl. Bogumil et al. 2006; Bouckaert 2006; Hood 1991; Naschold/Bogumil 2000; Pollitt/Bouckaert 2004). Wenngleich die konkrete nationale Ausgestaltung und Interpretation von NPM teilweise stark variiert, so besteht doch weitgehend Einigkeit darüber, dass diese Ent-

\footnotetext{
* Für hilfreiche Anregungen und Kritik bedanken wir uns bei den anonymen Gutachtern der PVS und der PVS-Redaktion.
} 
wicklung durch einen konvergierenden Trend gekennzeichnet ist: der Abkehr von klassischen bürokratischen Strukturen, wie sie idealtypisch von Max Weber identifiziert wurden, und der Hinwendung zu marktorientierten Prinzipien. Dieser globale Trend wurde in erheblichem Maße von internationalen Organisationen befördert, die als Diffusionsakteure und Diskussionsforen die Entwicklung und Ausgestaltung nationaler Verwaltungsreformen beeinflussten (Hood 1995; Lægreid 2002).

Interessanterweise wurden jedoch bislang entsprechende administrative Reformprozesse innerhalb dieser Organisationen selbst wissenschaftlich kaum untersucht. Im Zentrum der politikwissenschaftlichen Analyse internationaler Organisationen stehen deren Entwicklung und Kompetenzen sowie deren jeweilige Rolle als Arena für Verhandlungen zwischen ihren Mitgliedstaaten. Erst in jüngerer Zeit finden sich vereinzelt Untersuchungen, die internationale Organisationen als Verwaltungen konzipieren und in ihrer Rolle und Funktion als internationale Bürokratien beleuchten (Bauer/Knill 2007; Barnett/Finnemore 1999, 2004; Dijkzeul 1997; Dijkzeul/Beigbeder 2003; Hooghe 2001; Liese/Weinlich 2006; Reinalda/Verbeek 2004). Kurz: Es gibt weder fundierte Erkenntnisse darüber, ob internationale Organisationen die Prinzipien einer effizienten Verwaltung, die sie gegenüber ihren Mitgliedsstaaten einfordern, auch selbst umgesetzt haben, noch verfügen wir über ein hinreichendes theoretisches Instrumentarium, das differenzierte Aussagen über die Auslöser und die Dynamik von Veränderungsprozessen in diesen Organisationen ermöglicht.

Diese Fragen verweisen auf ein sehr weitreichendes Forschungsdefizit, das deutlich über den hier konkret betrachteten Untersuchungsgegenstand des Personalmanagements hinausgeht. So ist bislang völlig ungeklärt, ob und inwieweit internationale Organisationen in gleicher Weise wie Staaten oder nationale Organisationen für die Übernahme global zirkulierender Reformmodelle empfänglich sind. Ebenfalls gibt es keinerlei Informationen darüber, inwieweit internationale Organisationen, die als Diffusionsakteure und Fürsprecher globaler Reformmodelle auftreten, von diesen Modellen so überzeugt sind, dass sie diese auch selbst übernehmen. Mit anderen Worten: Sind internationale Organisationen lediglich Agenten transnationaler Politikdiffusion oder sind sie selbst Gegenstand und Ziel dieser Diffusionsprozesse? Ungeklärt ist überdies, wie jeweils beobachtbare Übernahmemuster auf der Ebene internationaler Organisationen theoretisch erklärt werden können. Die Analyse dieser Fragen erfordert es schließlich, dass auf diese Weise das politisch-administrative System - und damit das Innenleben internationaler Organisationen explizit zum Gegenstand politikwissenschaftlicher Forschung gemacht wird. Eine solche Perspektive, wie sie bislang etwa für die Untersuchung nationaler Bürokratien eingenommen wird, ist für die Ebene der internationalen Organisationen in der Politikwissenschaft nicht systematisch verfolgt worden.

Ziel dieses Artikels ist es, zum Abbau dieses Forschungsdefizits beizutragen. Zu diesem Zweck werden die Reformen des Personalmanagements in der Europäischen Kommission und im Sekretariat der OECD seit Mitte der 1990er Jahre vergleichend analysiert. Ungeachtet relativ ähnlicher Rahmenbedingungen weisen beide Organisationen in diesem Zeitraum große Unterschiede in der Reformentwicklung auf. Während in der Kommission sehr umfassende Verwaltungsreformen erfolgten (vgl. Bauer 2006; Cini 2002; Kassim 2004a, 2004b; Levy 2003; Pollitt/Bouckaert 2004), blieb die Reform des OECD-Sekretariats deutlich hinter den Standards zurück, die die OECD als 
Promotor von NPM und durch Veröffentlichungen ihres Public Management Komitees (PUMA) ${ }^{1}$ in den 1990 er Jahren als Orientierungspunkte für Verwaltungsreformen der westlichen Industriestaaten selbst erarbeitet hat (Sahlin-Andersson 2002; Stone 2004).

Diese Diskrepanz ist umso erstaunlicher, als sich beide Organisationen - trotz ihrer Unterschiede bezüglich ihrer Kompetenzen (supranational versus international) - in zentralen Eigenschaften gleichen. Beide Organisationen können als internationale Bürokratien charakterisiert werden, in denen Mitarbeiter mit sehr ähnlichem Bildungshintergrund beschäftigt sind. Beide Organisationen wurden Ende der 1950er Jahre gegründet und waren durch eine legalistische, klassisch-bürokratische Verwaltungstradition geprägt (Coombes 1970; Hahn/Weber 1976). Zudem werden beide Organisationen durch westliche Industriestaaten konstituiert. Westeuropäische Staaten sind in beiden Organisationen vertreten.

Im Zentrum der vorliegenden Analyse steht daher die Frage, wie - trotz ähnlicher Rahmenbedingungen - die unterschiedliche Reformentwicklung im Personalmanagement beider Organisationen erklärt werden kann. $\mathrm{Zu}$ diesem Zweck werden zunächst die empirischen Befunde für beide Organisationen vergleichend dargestellt. Hierzu wird ein Klassifikationsschema entwickelt, auf dessen Basis das Ausmaß und die Reichweite von Reformentwicklungen gemessen und verglichen werden können (Abschnitt 2). Im Zentrum des dritten Abschnitts steht die Frage der Erklärung dieser Ergebnisse. Wir zeigen, dass bestehende theoretische Ansätze nicht ausreichen, um die beobachteten Reformunterschiede zwischen beiden Organisationen zu verstehen. Hierzu bedarf es einer partiellen Modifikation bestehender Theorien, welche darauf abhebt, dass Organisationen nicht per se Reformmodelle übernehmen, die in ihrem Umfeld dominant sind. Vielmehr machen sie solche Übergangsentscheidungen von den relativen Legitimationsgewinnen abhängig, die mit Reformen verbunden sind. Letztere können in Abhängigkeit der jeweiligen Ausgangsbedingungen und Problemkonstellationen von Organisation zu Organisation variieren. In Abschnitt 4 werden die Erkenntnisse zusammengefasst und Anregungen für die weitere Forschung gegeben.

\section{Die Reform des Personalmanagements in der Europäischen Kommission und im OECD-Sekretariat}

Zentraler Gegenstand dieses Artikels ist die Analyse der Reform des Personalmanagements der Europäischen Kommission und des OECD-Sekretariats. Unter Personalmanagement verstehen wir die Bereiche Rekrutierung, Laufbahngestaltung, Personalbeurteilung und Personalentwicklung (Davies 2002; Vaanholt 1997). Wir betrachten somit nur am Rande die Personalpolitik, die als Rahmenbedingung für das Personalmanagement gilt und etwa den Sozialversicherungsschutz der Mitarbeiter, Maßnahmen im Bereich Gender Mainstreaming, Urlaubs- und Arbeitszeitregelungen oder Regelungen zur Entlassung bzw. Pensionierung umfasst (Reichard 2005).

1 PUMA wurde 2005 in Public Governance and Territorial Development (GOV) umbenannt. 
Um die Veränderungen unserer abhängigen Variablen zu messen, unterscheiden wir zwischen den Idealtypen von Max Webers Bürokratiemodell (BM) (vgl. Getz/Jüttner 1972; Henneberger 1997; Kieser 2002; McGregor/Solano 1996) und dem Leitbild NPM (vgl. Bouckaert 2006; Liebel/Oechsler 1992; Maguire 1993; OECD 1995, 2005a; Pollitt/Bouckaert 2004; Vaanholt 1997). Bei Letzterem handelt es sich zwar nicht um ein einheitliches „Reformrezept“, da NPM verschieden definiert, interpretiert und umgesetzt wird (Schedler/Proeller 2003). Im Bereich Personalmanagement zeichnet sich NPM aber durch seine universalen Kriterien Leistungsbezug, individuelle Anreizgestaltung und Flexibilität aus (Bouckaert 2006; Demmke 2006; Pollitt/Bouckaert 2004) und kann so als relevantes Modell für Reformen herangezogen werden (Naschold/Bogumil 2000: 84; OECD 2005a: 181).

Tabelle 1: Operationalisierung der abhängigen Variablen

\begin{tabular}{|c|c|c|}
\hline Indikatoren & Wert (BM) & Wert (NPM) \\
\hline \multicolumn{3}{|l|}{ Rekrutierung } \\
\hline Auswahlverfahren & $\begin{array}{l}\text { formale Voraussetzungen und } \\
\text { Nationalitätenproporz }\end{array}$ & $\begin{array}{l}\text { Leistungsprinzip und standardisierte } \\
\text { Auswahlmethoden }\end{array}$ \\
\hline $\begin{array}{l}\text { Vergabe von } \\
\text { Führungspositionen }\end{array}$ & $\begin{array}{l}\text { freiwillige Ausschreibung, } \\
\text { auf unbeschränkte Zeit }\end{array}$ & $\begin{array}{l}\text { Ausschreibung obligatorisch, } \\
\text { leistungsabhängige Dauer }\end{array}$ \\
\hline $\begin{array}{l}\text { Anforderungsprofil für } \\
\text { Führungskräfte }\end{array}$ & $\begin{array}{l}\text { Fachkenntnisse, kaum } \\
\text { Ressourcenverantwortung }\end{array}$ & $\begin{array}{l}\text { Managementkenntnisse, persönliche } \\
\text { Ressourcenverantwortung }\end{array}$ \\
\hline \multicolumn{3}{|l|}{ Laufbahngestaltung } \\
\hline Besetzung offener Stellen & Eingangsstufe einer Laufbahn & jede Position kann besetzt werden \\
\hline Laufbahnstruktur & horizontal und undurchlässig & vertikal und durchlässig \\
\hline Grundgehalt & abhängig von Besoldungsgruppe & an Aufgabe angepasst \\
\hline Gehaltszulage & $\begin{array}{l}\text { automatisch und linear, } \\
\text { vom Dienstalter abhängig }\end{array}$ & $\begin{array}{l}\text { leistungsorientierte Bezahlung für } \\
\text { alle Mitarbeiter }\end{array}$ \\
\hline Individuelle Belohnung & keine & Cafeteria-Systeme \\
\hline \multicolumn{3}{|l|}{ Personalbeurteilung } \\
\hline Leistungsbeurteilung & $\begin{array}{l}\text { nicht obligatorisch und selten } \\
\text { durchgeführt }\end{array}$ & $\begin{array}{l}\text { verpflichtende jährliche } \\
\text { Zielvereinbarung für alle Mitarbeiter }\end{array}$ \\
\hline $\begin{array}{l}\text { Potenzialbeurteilung } \\
\text { (zukünftige Eignung) }\end{array}$ & $\begin{array}{l}\text { nicht obligatorisch und parallel zur } \\
\text { Leistungsbeurteilung durchgeführt }\end{array}$ & $\begin{array}{l}\text { obligatorisch für alle Mitarbeiter } \\
\text { und Durchführung unabhängig von } \\
\text { der Leistungsbeurteilung }\end{array}$ \\
\hline Vorgesetztenbeurteilung & keine & $\begin{array}{l}\text { für alle Vorgesetzten, regelmäßige } \\
\text { Durchführung }\end{array}$ \\
\hline Ergebnisverwertung & $\begin{array}{l}\text { keine Verknüpfung mit Beförderung } \\
\text { und Gehaltszulagen }\end{array}$ & $\begin{array}{l}\text { direkte Verknüpfung mit } \\
\text { Beförderung und Gehaltszulagen }\end{array}$ \\
\hline \multicolumn{3}{|l|}{ Personalentwicklung } \\
\hline $\begin{array}{l}\text { Ausgaben und } \\
\text { Möglichkeiten für Fort- } \\
\text { und Weiterbildung }\end{array}$ & konstant und niedrig & tendenziell steigend und angemessen \\
\hline Strategische Ausrichtung & keine strategische Ausrichtung & Strategie des lebenslangen Lernens \\
\hline Führungskräfteentwicklung & $\begin{array}{l}\text { nicht verpflichtend und kaum } \\
\text { Angebote }\end{array}$ & $\begin{array}{l}\text { verpflichtend und eine Vielzahl an } \\
\text { Angeboten }\end{array}$ \\
\hline
\end{tabular}

Quelle: Eigene Darstellung (basierend auf Naschold/Bogumil 2000; Pollitt/Bouckaert 2004; Vaanholt 1997). 
Das Ausmaß der Übereinstimmung der jeweiligen Indikatoren mit den beiden Idealtypen messen wir anhand einer Ordinalskala mit fünf Kategorien (0 bis 4). Damit soll der Tatsache Rechnung getragen werden, dass empirisch oftmals Ausprägungen zwischen diesen Idealtypen anzutreffen sind. Der Wert 0 bedeutet, dass die Ausprägung des Indikators vollständig mit den Ausprägungen des Bürokratiemodells übereinstimmt; der Wert 4 impliziert vollständige Übereinstimmung mit NPM. Ein Wert von 1 bezeichnet Konstellationen, in denen die Ausprägungen eher dem BM als dem NPM entsprechen. So würde z. B. eine Personalbeurteilung mit 1 codiert, wenn sie zwar obligatorisch, jedoch nur selten und lediglich für einen geringen Teil der Belegschaft durchgeführt wird. Der Wert 2 bezieht sich auf die Fälle, in denen die empirische Ausprägung in etwa zwischen den Idealtypen liegt; der Wert 3 impliziert eine größere Nähe zum NPM als zum BM. Dem Wert 3 würde z. B. eine leistungsorientierte Bezahlung zugeordnet, die einen nennenswerten Teil des Gehalts darstellt und als monetärer Anreiz für viele, jedoch nicht alle Mitarbeiter eingesetzt wird. $\mathrm{Zu}$ betonen ist hierbei grundsätzlich, dass mit dieser Klassifizierung keine normativen Annahmen im Hinblick auf die Leistungsfähigkeit beider Modelle verbunden sind. Vielmehr stehen die Beschreibung der Reformentwicklungen und deren Erklärung im Mittelpunkt der vorliegenden Analyse.

Die Europäische Kommission und das OECD-Sekretariat regeln ihr Personalmanagement in ihren Personalstatuten („staff rules“) und den dazugehörigen Durchführungsbestimmungen („staff regulations“). Das Personalstatut kann nur mit Zustimmung der Mitgliedsstaaten verändert werden, die im jeweiligen Rat der beiden Organisationen vertreten sind. Die Durchführungsbestimmungen können hingegen bei der OECD vom Generalsekretär und bei der Kommission vom Kollegium der Kommissare allein verändert werden.

Im Folgenden betrachten wir alle Veränderungen dieser formalen Regeln seit Mitte der 1990er Jahre (1995 bis 2007). Die Wahl des Untersuchungszeitraumss orientiert sich dabei an der Tatsache, dass ab Mitte der 1990er Jahre von einer Etablierung von NPM als global dominantem Reformmodell für administrative Reformen ausgegangen werden kann (Naschold/Bogumil 2000). Überdies waren in dem davor liegenden Zeitraum weder in der Europäischen Kommission noch im OECD-Sekretariat nennenswerte Reformen im Bereich des Personalmanagements zu verzeichnen. Die Erhebung der relevanten Daten stützt sich neben der Auswertung der Sekundärliteratur auf die Analyse von Dokumenten der untersuchten Organisationen sowie auf 15 halbstandardisierte Forschungsinterviews, die mit einschlägigen Funktionsträgern in beiden Organisationen im Zeitraum von Januar bis Juni 2006 durchgeführt wurden.

\subsection{Die Reform des Personalmanagements in der Europäischen Kommission}

Die Regeln des Personalstatuts und der Durchführungsbestimmungen in der Europäischen Kommission (Europäische Kommission 2004a, 2004b) richten sich an ca. 23000 Mitarbeiter, die zum größten Teil über Verträge auf Lebenszeit verfügen. Daneben bestehen noch Beschäftigungsverhältnisse mit 7000 sonstigen Bediensteten, wie etwa Hilfskräften, Bediensteten auf Zeit und abgeordneten nationalen Sachverständigen. Für diese Gruppe gelten Sonderregelungen, die an dieser Stelle nicht berücksich- 
tigt werden. Insgesamt zeigen sich im Untersuchungszeitraum sehr weitreichende Reformen des Personalmanagements, die sich auf nahezu alle Teilbereiche erstrecken.

Mitte der 1990er Jahre war das Personalmanagement der Kommission noch weitgehend in der Nähe des BM-Ideals zu verorten. Dies wird zunächst bezüglich der Dimension der Rekrutierung deutlich. Bei der Vergabe von Führungspositionen und dem Anforderungsprofil an das Führungspersonal bestand eine vollkommene Übereinstimmung mit BM-Merkmalen. Führungspositionen mussten intern nicht ausgeschrieben werden und ihre Besetzung wurde in hohem Maße durch die Kommissare, ihre Kabinette und die Mitgliedsstaaten bestimmt (Lequesne 1996: 405). Das Anforderungsprofil für Positionen von Generaldirektoren und ihren Stellvertretern war formal kaum umrissen und beruhte hauptsächlich auf ihrer Fachkompetenz (Bauer 2008; Wille 2007: 39 f.). Lediglich das Auswahlverfahren kombinierte in gleicher Weise Elemente von BM und NPM. So werden seit Gründung der Europäischen Kommission die Beamten über den sogenannten „concours“ rekrutiert, bei dem Kandidaten nach Leistung und Nationalitätenproporz ausgewählt werden (Getz/Jüttner 1972; Stevens/Stevens 2001). Dieses standardisierte Auswahlverfahren wurde jedoch teilweise dadurch umgangen, dass Kabinettsmitglieder der Kommissare bzw. Mitarbeiter mit Zeitverträgen ohne weitere Prüfung ihrer Leistung eine Anstellung auf Lebenszeit erhielten.

Auch die Laufbahngestaltung bewegte sich nahezu vollkommen entlang des BMIdeals. Die Karrierestruktur der Kommission war durch vier Laufbahngruppen (A/LA, B, C, D) mit jeweils vier bis acht Besoldungsgruppen (z. B. A1, A2, A3 ...) gekennzeichnet. Die Besetzung offener Stellen erfolgte dabei - mit Ausnahme der Spitzenpositionen - auf der Eingangsstufe einer Laufbahngruppe. Die Aufstiegsmöglichkeiten innerhalb der Laufbahngruppen waren relativ begrenzt und der Übergang von einer Laufbahngruppe zur nächst höheren sehr schwer zu realisieren (Stevens/Stevens 2001: 98). Es war daher keine Seltenheit, dass Beamte bereits nach 15 bis 20 Dienstjahren die höchste Besoldungsgruppe erreichten. Die Posten von Generaldirektoren und ihren Stellvertretern (A1 und A2) galten z. B. nicht als üblicher Bestandteil einer Beamtenkarriere, sondern wurden oft aus politischen Beweggründen besetzt (Coombes 1970: 146; Stevens/Stevens 2001: 74). Das Grundgehalt der Beamten war an ihre Stelle bzw. Besoldungsgruppe gebunden und nicht an die Aufgabe oder Verantwortung, die sie de facto übernommen hatten. Die Besoldungsgruppen hatten zudem bis zu acht Dienstaltersstufen, die jedem Beamten alle zwei Jahre eine automatische Steigerung der Bezüge um 4,5 Prozent garantierte. Gehaltszulagen, die sich explizit nach der Leistung eines Beamten richten, gab es nicht.

Die Systeme der Personalbeurteilung und der Personalentwicklung waren ebenfalls eindeutig am BM ausgerichtet. So fanden etwa Leistungsbeurteilungen nur in unregelmäßigen Abständen statt und wirkten sich kaum auf Beförderungschancen aus (Interview, 12.05.2006). Die Leistungsbeurteilung basierte auf einer undifferenzierten fünfteiligen Skala, bei der die inflationäre Vergabe der Note "gut" dazu führte, dass keine tatsächliche Unterscheidung der Mitarbeiter nach Leistung mehr stattfinden konnte (Stevens/Stevens 2001: 100 f.). 
Tabelle 2: Die Reform des Personalmanagements in der Europäischen Kommission

\begin{tabular}{|l|c|c|l|}
\hline Indikatoren & 1995 & 2007 & Veränderung \\
\hline Rekrutierung & 2 & 2 & keine Veränderung \\
Auswahlverfahren & 0 & 1 & Richtung NPM \\
Vergabe von Führungspositionen & 0 & 2 & Richtung NPM \\
Anforderungsprofil für Führungskräfte & & & \\
\hline Laufbahngestaltung & 1 & 2 & Richtung NPM \\
Besetzung offener Stellen & 0 & 3 & Richtung NPM \\
Laufbahnstruktur & 0 & 1 & Richtung NPM \\
Grundgehalt & 0 & 2 & Richtung NPM \\
Gehaltszulage & 0 & 0 & keine Veränderung \\
Individuelle Belohnung & 1 & & \\
\hline Personalbeurteilung & 0 & 4 & Richtung NPM \\
Leistungsbeurteilung & 0 & 1 & Richtung NPM \\
Potenzialbeurteilung NPM \\
Vorgesetztenbeurteilung & 0 & 2 & Richtung NPM \\
Ergebnisverwertung & & & \\
\hline Personalentwicklung & 0 & 4 & Richtung NPM \\
Maßnahmen der Fort- und Weiterbildung & 0 & 4 & Richtung NPM \\
Strategische Ausrichtung & 0 & 4 & Richtung NPM \\
Führungskräfteentwicklung & & 4 & \\
\hline
\end{tabular}

Quelle: Eigene Darstellung.

Eine Beförderung erfolgte so in der Regel nach einer bestimmten Dienstzeit automatisch. ${ }^{2}$ Schließlich zeichneten sich die Maßnahmen der Fort- und Weiterbildung Ende der 1990er Jahre durch rückläufige Ausgaben, ein geringes Budget und lediglich zweieinhalb Fortbildungstage pro Jahr und Mitarbeiter aus (Stevens/Stevens 2001: 106). Sowohl für die Führungskräfteentwicklung als auch für die Angebote der Fort- und Weiterbildung insgesamt gab es kein strategisches Konzept (Interview, 12.05.2006).

Betrachtet man nun die aktuelle Konstellation, so wird deutlich, dass sich die Ausprägungen der Variable Personalmanagement von 1995 bis 2007 nahezu alle in Richtung NPM verändert haben (vgl. Tabelle 2). Im Bereich Rekrutierung verbleiben diese Verschiebungen allerdings auf eher moderatem Niveau und finden sich insbesondere bei der Vergabe von Führungspositionen und dem Anforderungsprofil an Führungskräfte wieder. So werden alle Führungspositionen nun intern ausgeschrieben und durch ein formalisiertes Auswahlverfahren unter Einbeziehung des „Comité Consultatif des Nominations“ (CCN) ausgewählt (Egeberg 2006: 39f.). Hierdurch wird der Einfluss von Kommissaren und Mitgliedsstaaten auf die Besetzung dieser Posten erheblich reduziert. Eine zusätzliche externe Ausschreibung bleibt allerdings die Ausnahme. Die Ernennung bzw. Beförderung zum Generaldirektor oder Referatsleiter (mittleres Management) setzt neben Fachkenntnissen nun auch Managementkenntnisse voraus (Interview, 12.05.2006). Kompetenzen wie Führungsqualitäten und Kommunikationsstärke werden für neu rekrutierte Führungskräfte sogar im Rahmen eines „Assessment Cen-

2 In einer Ende der 1990er Jahre erfolgten Befragung über die tatsächlich relevanten Entscheidungskriterien für Beförderungen antworteten die Beamten in folgender Reihenfolge: „Vitamin B“, Länge der Dienstzugehörigkeit, Nationalität und Glück (Davies 2002: 178). Bei der Umfrage sagten 52 Prozent ebenfalls: „It doesn't matter what I do, promotion won't come any faster" (Spence 1997: 75). 
ters" überprüft. Eine persönliche Ressourcenverantwortung der Manager besteht aufgrund ihrer Verträge auf Lebenszeit allerdings weiterhin nur eingeschränkt. Seit der Einführung eines ergebnisorientierten Managements muss die Aufgabenerledigung aber transparent dargestellt werden und der Generaldirektor gegenüber dem Europäischen Parlament Rechenschaft über sein Arbeitsprogramm ablegen.

Noch deutlicher ist die Bewegung in Richtung NPM bei der Laufbahngestaltung. So führte die Kommission ein neues Laufbahnsystem ein, das nur noch auf zwei Gruppen beruht (Europäische Kommission 2004a, 2004b). Die Laufbahnen B und C wurden nach einer Übergangsregelung in die Funktionsgruppe „Assistenz“ (AST 1-11) und die Laufbahnen A und LA in die Funktionsgruppe „Administration“ (AD 5-16) überführt. Die Laufbahngruppe D wurde abgeschafft, da die anfallenden Aufgaben (vor allem technische Dienste) fortan nur noch von Vertragsbediensteten erledigt werden. Durch die höhere Anzahl der Besoldungsgruppen ergeben sich für die Beamten nun deutlich mehr Aufstiegsmöglichkeiten. Das Grundgehalt orientiert sich weiterhin an der Besoldungsgruppe und damit (nicht unbedingt) an der zu verrichtenden Aufgabe. Immerhin wurde das Grundgehalt für jene Beamten, die finanzielle und personelle Verantwortung tragen, um eine Dienstaltersstufe aufgestockt. Es besteht jedoch weiterhin die Möglichkeit, dass ein Beamter trotz seiner Leistung insgesamt weniger verdient als sein Assistent, nur weil er kürzer im Amt ist. Diese Überschneidungen wurden jedoch im Vergleich zu den 1990er Jahren sowohl durch mehr Besoldungsgruppen (16 für AD und AST anstelle von vormals acht in Laufbahn A bzw. fünf in Laufbahn B und C) als auch durch weniger Dienstalterstufen (fünf anstelle von acht) reduziert (Interview, 12.05.2006). Das Erreichen der nächsten Dienstaltersstufe ist zwar weiterhin unabhängig von der erbrachten Leistung, d. h. es gibt keine leistungsorientierte Bezahlung. Das Durchlaufen der nun fünf statt acht Dienstaltersstufen ist aber nicht mehr mit einem gleichmäßigen Gehaltsanstieg verbunden, sondern wird degressiv gestaltet.

Die größten Veränderungen vollzogen sich bei der Personalbeurteilung und der Personalentwicklung. In diesen Bereichen ist die Kommission bei fast allen Indikatoren vollständig auf NPM übergegangen. So wurde die Personalbeurteilung umfassend reformiert. Im neuen „System der Begleitung der beruflichen Entwicklung" wird die Leistung eines jeden Beamten jährlich und auf der Grundlage einer Arbeitsplatzbeschreibung und Zielvereinbarung mit dem Vorgesetzten beurteilt. Außerdem wird nun für alle Mitarbeiter verbindlich eine Potenzialbeurteilung durchgeführt, die unabhängig von der zurückliegenden Leistungsbeurteilung die mittel- bis langfristigen Entwicklungschancen der Mitarbeiter aufzeigen soll. Die Vorgesetztenbeurteilung befindet sich hingegen noch in einer Pilotphase. Um die Ergebnisverwertung der Personalbeurteilungen zu verbessern und Leistungen besser vergleichbar zu machen, wurde ein neuer, differenzierter Kriterienkatalog mit bis zu 32 "Verdienst- und Prioritätspunkten“ eingeführt. Ein Beamter kann nun „Punkte“ auf seinem Konto ansammeln und für zukünftige Beförderungsstellen einsetzen. Beamte, die über der sich am Haushaltsplan orientierenden "Schwelle“ liegen, werden dann - aufgrund ihrer Leistung - automatisch befördert. Für Beamte mit durchschnittlichen Leistungen wird allerdings auch in $\mathrm{Zu}$ kunft eine vom Dienstalter abhängige automatische Beförderung erfolgen.

Mit der Entscheidung für eine Reform der Personalentwicklung wurde bereits im Jahre 2001 das Fortbildungsbudget um 63,6 Prozent erhöht und steigt seitdem konti- 
nuierlich an (Europäische Kommission 2005). Im Jahre 2002 nahm ein EU-Beamter im Durchschnitt 6,9 Fortbildungstage pro Jahr wahr; die aktuelle Zahl liegt bei elf Tagen. Mit der Reform verbunden war zudem eine strategische Neuausrichtung der Weiterbildungspolitik - hin zu einer Kultur des lebenslangen Lernens. Die Führungskräfteentwicklung, d. h. die Fortbildung von Managern, die Verantwortung für personelle und finanzielle Ressourcen übernehmen, ist seit der Reform verpflichtend.

Zusammenfassend lässt sich festhalten, dass die Ausprägungen des Personalmanagements Mitte der 1990er Jahre noch fast vollständig dem Idealtyp des Bürokratiemodells entsprachen. Seit der Reform im Jahre 2004 befindet sich das Personalmanagement der Kommission jedoch in vielen Bereichen relativ nahe am Idealtyp des NPM. Dies gilt insbesondere für die Personalentwicklung, aber auch für die Laufbahngestaltung und die Personalbeurteilung. Weniger stark wurden dagegen die Bereiche Rekrutierung und Anreizgestaltung reformiert.

\subsection{Die Reform des Personalmanagements im OECD-Sekretariat}

Die Regeln des aktuellen Personalstatuts der OECD richten sich an ca. 2000 Mitarbeiter („Officials“) des Sekretariats, die fast alle in Paris arbeiten und entweder über befristete oder unbefristete Verträge verfügen. Daneben sind 300 kurzfristig Beschäftigte wie Berater, Trainees und Hilfskräfte tätig. Für diese Gruppe bestehen Sonderregelungen, die an dieser Stelle nicht weiter berücksichtigt werden. Insgesamt sind im Untersuchungszeitraum nur wenige Veränderungen des Personalmanagements zu verzeichnen.

Zum Ausgangszeitpunkt der Untersuchung Mitte der 1990er Jahre war der Bereich Rekrutierung mehr oder weniger zwischen BM und NPM zu verorten. Das Auswahlverfahren bei der Rekrutierung neuer Mitarbeiter stützte sich primär auf das Leistungsprinzip (Interview, 14.03.2006). Zwar sollten Mitarbeiter aus jedem Mitgliedsstaat angemessen vertreten sein („national balance“), doch gab es kein institutionalisiertes Gremium, das die Einhaltung dieses Ziels kontrollierte. Die Einhaltung des Leistungsprinzips wurde in der Regel durch ein standardisiertes Auswahlverfahren gewährleistet. Die Rekrutierung von Mitarbeitern für Projektstellen, die aus freiwilligen Beiträgen der Mitgliedsstaaten finanziert wurden, erfolgte jedoch oft unstandardisiert. Es lag im Ermessen des jeweiligen Direktorats, ob die Stelle ausgeschrieben wurde und ob die Bewerber das normale Auswahlverfahren durchlaufen mussten. Auch Führungspositionen wurden nicht obligatorisch ausgeschrieben. Die Entscheidung darüber war dem Generalsekretär vorbehalten.

Bei der Laufbahngestaltung entsprach lediglich das Verfahren der Besetzung offener Stellen dem NPM - ein Quereinstieg in die Laufbahn des OECD-Sekretariats ist üblich, da für jede offene Stelle der jeweils passende Bewerber ausgewählt wird. Für alle anderen Indikatoren lagen die Werte nahezu vollständig auf der BM-Position. Die Laufbahnstruktur wurde etwa seit Gründung der OECD kaum verändert. Sie gliedert sich in die Laufbahnen A, L, B und C und die dazugehörigen Besoldungsgruppen (OECD 2007). Die Laufbahn A umfasst 45 Prozent des Personals und ist diejenige, die in der Hierarchie am höchsten angesiedelt ist, vergleichbar dem höheren Dienst in Deutschland. Ihr folgen die Laufbahnen B (Mitarbeiter mit Unterstützungs-, Statistik- 
und Verwaltungsaufgaben) und C (technische und handwerkliche Dienste). Für etwa 80 Dolmetscher ist eine eigene Laufbahn (L) vorgesehen. Die Laufbahnstruktur ist insgesamt relativ undurchlässig und bietet nur wenige Aufstiegsmöglichkeiten (Interview, 17.03.2006). Seit Einführung der ergebnisorientierten Budgetierung wird es außerdem zunehmend schwierig, Mitarbeiter flexibel einzusetzen und sie entsprechend der Erfüllung ihrer (neuen) Aufgabe zu entlohnen. Einige Mitgliedsstaaten sind sogar der Ansicht, dass das starre Karrieresystem die OECD daran hindert, jene Spezialisten zu rekrutieren, die sie eigentlich für die Erfüllung ihrer Aufgaben bräuchte (Interview, 14.03.2006).

Tabelle 3: Die Reform des Personalmanagements im OECD-Sekretariat

\begin{tabular}{|l|c|c|l|}
\hline Indikatoren & 1995 & 2007 & Veränderung \\
\hline Rekrutierung & 3 & 3 & keine Veränderung \\
Auswahlverfahren & 1 & 3 & Richtung NPM \\
Vergabe von Führungspositionen & 1 & 3 & Richtung NPM \\
Anforderungsprofil für Führungskräfte & & 4 & keine Veränderung \\
\hline Laufbahngestaltung & 4 & 0 & keine Veränderung \\
Besetzung offener Stellen & 0 & 0 & keine Veränderung \\
Laufbahnstruktur & 0 & 1 & keine Veränderung \\
Grundgehalt & 1 & 1 & keine Veränderung \\
Gehaltszulage & 1 & & \\
Individuelle Belohnung & 1 & 4 & Richtung NPM \\
\hline Personalbeurteilung & 0 & 4 & Richtung NPM \\
Leistungsbeurteilung & 0 & 1 & Richtung NPM \\
Potenzialbeurteilung & 1 & 1 & keine Veränderung \\
Vorgesetztenbeurteilung & 1 & 0 & Richtung BM \\
Ergebnisverwertung & 1 & 1 & keine Veränderung \\
\hline Personalentwicklung & 1 & 1 & keine Veränderung \\
\hline Maßnahmen der Fort- und Weiterbildung & & & \\
Strategische Ausrichtung & & & \\
Führungskräfteentwicklung & &
\end{tabular}

Quelle: Eigene Darstellung.

Das Grundgehalt eines Mitarbeiters richtet sich nach der jeweiligen Besoldungsgruppe der Laufbahn, und die Gehaltszulage besteht aus den jeweiligen Dienstaltersstufen („steps“), die alle zwei Jahre progressiv gewährt werden. Nur bei besonders schlechten Leistungen kann ihre Auszahlung für einen kurzen Zeitraum ausgesetzt werden (Interview, 17.05.2006). Die höchsten Besoldungsgruppen A7 und A6 verfügen seit einer Erhöhung im Jahre 1999 über sechs bzw. acht Dienstaltersstufen, die restlichen Besoldungsgruppen in der Regel über zehn oder elf. Dies führt in Verbindung mit dem Grundgehalt zu einer Überlappung der Besoldungsgruppen, sodass ein Vorgesetzter trotz höherer Qualifikation und Verantwortung weniger verdienen kann als sein Untergebener. Die Bezahlung richtet sich damit nach dem Senioritätsprinzip; eine leistungsorientierte Bezahlung ist nicht vorgesehen (Interview, 14.03.2006). Der Generalsekretär kann aber Mitarbeiter seiner Wahl für besondere Leistungen mit zusätzlichen Dienstaltersstufen auszeichnen (individuelle Belohnung).

Was die Dimensionen der Personalbeurteilung und Personalentwicklung anbelangt, so bewegte sich das OECD-Sekretariat ebenfalls sehr stark entlang des Bürokratiemo- 
dells. So erfolgten Personal- und Potenzialbeurteilungen auf einer sehr unsystematischen Basis. Gleiches galt für die Fort- und Weiterbildung sowie für die Führungskräfteentwicklung. Letztere waren nicht verpflichtend und stellten nur sehr wenige Angebote bereit (Interview, 14.03.2006).

Vergleicht man diesen Ausgangszustand mit der aktuellen Ausgestaltung des Personalmanagements, so zeigen sich nur wenige Veränderungen. So blieb die Konstellation in den Bereichen Laufbahngestaltung und Personalentwicklung weitgehend konstant. Bei der Personalentwicklung zeigte sich sogar eine schwache Bewegung in Richtung BM (also ein gegenläufiger Trend, wenn man die globale Reformtendenz in Richtung NPM betrachtet). So sind hier die Trainingsstunden pro Mitarbeiter und das Budget für Training insgesamt im Untersuchungszeitraum deutlich zurückgegangen (Interview, 15.03.2006). ${ }^{3}$ Zurzeit überdenkt die OECD die Ausgestaltung ihrer Fort- und Weiterbildungsmaßnahmen; bis dato wird aber keine Strategie des lebenslangen Lernens verfolgt.

Moderate Verschiebungen in Richtung NPM finden sich allerdings im Bereich Personalrekrutierung. Seit 1999 erhalten Führungskräfte nur noch befristete Verträge, die bei guter Leistung verlängert werden können. Zudem erfolgen seitdem interne Besetzungen von Führungspositionen nur noch dann, wenn die infrage kommenden Mitarbeiter auf ihren unbefristeten Vertrag verzichten, d. h. in ein befristetes Arbeitsverhältnis überwechseln. Das Anforderungsprofil für Führungskräfte des OECD-Sekretariats beruht nun auf Fach- und Managementkenntnissen, da diese seit Einführung des ergebnisorientierten Managements einer starken „performance discipline“ unterliegen, die mit der Erfüllung bestimmter „outputs“ des Arbeitsprogramms verbunden ist (Interview, 15.03.2006).

Auch die Personalbeurteilung wurde im Jahre 1999 in Richtung NPM reformiert (Interview, 15.03.2006). Die Leistungsbeurteilung wird nun jährlich, für alle Mitarbeiter und auf der Grundlage einer vorangegangen Zielvereinbarung durchgeführt, und auch die Potenzialbeurteilung entspricht nun dem Idealtypus des NPM. Vorgesetztenbeurteilungen können in den Direktoraten optional (360-Grad-Feedback) durchgeführt werden und werden zur Weiterentwicklung der Führungskräfte genutzt (Interview, 15.03.2006). Prinzipiell sollen die Personalbeurteilungen Grundlage für Entscheidungen über Beförderungen und Vertragsverlängerungen sein, jedoch fehlt es an einem differenzierten Kriterienkatalog, der valide Informationen über die Leistung der Mitarbeiter zulässt (Interview, 14.03.2006). Beurteilungen haben somit fast keine formalisierte Wirkung auf Anreizgestaltung und Beförderung (Interview, 17.03.2006).

Zusammenfassend zeigt sich, dass das Personalmanagement des OECD-Sekretariats im Untersuchungszeitraum kaum reformiert wurde. Lediglich in den Bereichen Rekrutierung und Personalbeurteilung hat es nennenswerte Reformen gegeben. Die Laufbahngestaltung wurde nicht reformiert und wird auch seitens organisationsinterner Akteure als unzureichend und nicht mehr zeitgemäß eingestuft (Interview, 14.03.2006).

3 Aufgrund der guten Zusammenarbeit mit dem OECD-Sekretariat konnten für die Beurteilung der Reichweite der Reform genaue Angaben über das Budget für Weiterbildung, die Trainingsstunden pro Mitarbeiter und die Führungskräfteentwicklung verwertet werden (vgl. Tabelle 3). Diese Angaben sind jedoch, wie große Teile des Personalstatuts der OECD, nicht öffentlich zugänglich. 
Bei der Personalentwicklung muss aufgrund sinkender Ausgaben, der geringen Zahl von Trainingsstunden pro Mitarbeiter und der mangelnden strategischen Ausrichtung sogar von einer Bewegung hin zum Bürokratiemodell gesprochen werden.

\subsection{Die Reformen im Vergleich}

Ein Vergleich des Personalmanagements innerhalb des OECD-Sekretariats und der Europäischen Kommission zeigt, dass im Zeitablauf eine klare Verschiebung aufgetreten ist, was die jeweilige Nähe der Ausprägungen zum globalen Reformmodell des NPM betrifft. Lediglich im Bereich der Rekrutierung liegt das OECD-Sekretariat näher am NPM, da seine Führungskräfte im Gegensatz zur Europäischen Kommission nur über leistungsabhängige und befristete Verträge verfügen. Zum anderen wird das „merit principle“ bei der Rekrutierung stärker berücksichtigt als bei der Kommission. Die Laufbahngestaltung hat sich in beiden Organisationen insgesamt wenig in Richtung NPM bewegt, wobei allerdings für die Kommission eine vergleichsweise größere Reformbewegung zu verzeichnen ist. Dies liegt vor allem an der Reform der Laufbahnstruktur, die jetzt nur noch zwei Laufbahnen und mehr Aufstiegsmöglichkeiten vorsieht. Außerdem erhalten Manager für ihre besondere Aufgabe einen Zuschlag zum Grundgehalt und es gibt weniger stark ausgeprägte Überlappungen der Besoldungsgruppen.

Beide Organisationen haben ihre Leistungs- und Potenzialbeurteilung dem NPM angepasst und führen optional Vorgesetztenbeurteilungen durch. Ein wesentlicher Unterschied besteht aber darin, dass die Beamten der Kommission mit konstant guten Leistungen $\mathrm{ab}$ einer bestimmen Schwelle automatisch befördert werden. Die Personalbeurteilungen im OECD-Sekretariat haben hingegen keine hinreichend formalisierten

Tabelle 4: Die Reformen des Personalmanagements im Vergleich

\begin{tabular}{|l|ccc|ccc|}
\hline Indikatoren & $\begin{array}{c}\text { KOM } \\
(1995)\end{array}$ & $\begin{array}{c}\text { OECD } \\
(\mathbf{1 9 9 5 )}\end{array}$ & $\begin{array}{c}\text { Näher } \\
\text { an NPM }\end{array}$ & $\begin{array}{c}\text { KOM } \\
(2007)\end{array}$ & $\begin{array}{c}\text { OECD } \\
(2007)\end{array}$ & $\begin{array}{c}\text { Näher } \\
\text { an NPM }\end{array}$ \\
\hline Rekrutierung & 2 & 3 & OECD & 2 & 3 & OECD \\
Auswahlverfahren & 0 & 1 & OECD & 1 & 3 & OECD \\
Vergabe von Führungspositionen & 0 & 1 & OECD & 2 & 3 & OECD \\
Anforderungsprofil für Führungskräfte & 0 & & & & & \\
\hline Laufbahngestaltung & 1 & 4 & OECD & 1 & 4 & OECD \\
Besetzung offener Stellen & 0 & 0 & - & 3 & 0 & KOM \\
Laufbahnstruktur & 0 & 0 & - & 1 & 0 & KOM \\
Grundgehalt & 0 & 1 & OECD & 2 & 1 & KOM \\
Gehaltszulage & 0 & 1 & OECD & 0 & 1 & OECD \\
Individuelle Belohnung & & & & & & \\
\hline Personalbeurteilung & 1 & 1 & - & 4 & 4 & - \\
Leistungsbeurteilung & 0 & 0 & - & 4 & 4 & - \\
Potenzialbeurteilung & 0 & 0 & - & 1 & 1 & - \\
Vorgesetztenbeurteilung & 0 & 1 & OECD & 2 & 1 & KOM \\
Ergebnisverwertung & & & & & & KOM \\
\hline Personalentwicklung & 0 & 1 & OECD & 4 & 0 & KOM \\
Fort- und Weiterbildung & 0 & 1 & OECD & 4 & 1 & KOM \\
Strategische Ausrichtung & 0 & 1 & OECD & 4 & 1 & \\
Führungskräfteentwicklung & & &
\end{tabular}

Quelle: Eigene Darstellung. 
Auswirkungen auf Beförderung und Anreizgestaltung. Die Personalentwicklung zeigt schließlich am deutlichsten die Unterschiede auf: Die Europäische Kommission hat ihre Ausgaben für Weiterbildung stetig erhöht, gewährleistet mehr als zehn jährliche Fortbildungstage pro Mitarbeiter, verfolgt eine Strategie des lebenslangen Lernens und hat die Managementfortbildung von Führungskräften verpflichtend eingeführt.

Insgesamt lässt sich somit festhalten, dass wir seit Mitte der 1990er Jahre auf der Ebene der Europäischen Kommission sehr weitreichende Reformen des Personalmanagements beobachten können, welche in vielen Bereichen sehr nahe an den Idealtypus des NPM heranreichen. Im OECD-Sekretariat hingegen sind solche umfassenden Entwicklungen ausgeblieben. 1995 wies dessen Personalmanagement zwar noch eine größere Nähe zum NPM auf, jedoch hat sich diese Konstellation im Laufe der vergangenen Jahre zugunsten der Europäischen Kommission umgekehrt. Dieser Befund ist umso überraschender, als insbesondere die OECD international als prominenter Verfechter von Konzepten des NPM gilt. Im Folgenden wollen wir uns daher der Frage zuwenden, wie diese Entwicklung theoretisch erklärt werden könnte.

\section{Die Reformentwicklungen aus theoretischer Perspektive}

Bei der Suche nach Erklärungen für den Wandel von Organisationen lassen sich auf einer generellen Ebene endogene und exogene Faktoren unterschieden. Endogene Faktoren beziehen sich auf Eigenschaften und Merkmale der jeweiligen Organisation, während exogene Faktoren mögliche Einflüsse betreffen, die sich aus dem Umfeld der Organisation ergeben.

Als endogene Faktoren kommen in unserem Zusammenhang insbesondere interner Problemdruck (der sich aus der Perzeption und Politisierung von Performanzdefiziten ergibt) sowie die jeweiligen organisationsinternen Entscheidungsverfahren und -strukturen infrage, um die beobachtete Varianz in der Reformentwicklung beider Organisationen zu erklären. Eine nähere Betrachtung zeigt allerdings, dass zwischen OECD-Sekretariat und Kommission im Hinblick auf diese Aspekte keine entscheidenden Unterschiede bestehen. Beide waren Ende der 1990er Jahre mit internen Korruptionsvorwürfen bzw. Vorwürfen der intransparenten Rechnungslegung und damit mit einem Problemdruck konfrontiert (Peterson 2004), der in der Literatur als wichtiger Wirkungsmechanismus für Verwaltungsreformen gilt (Bauer 2006; Cini 2002; Geri 2001; Peterson 2004; Pollitt/Bouckaert 2004: 234). Da die Human-Resources-Abteilung der OECD die Notwendigkeit einer Reform des Personalmanagements erkannte und seit geraumer Zeit Lösungsansätze erarbeitet (OECD 1999: 32, 2001: 97, 2003: 94, 2006b: 102 f.), kann auch nicht von einer mangelnden Problemwahrnehmung relevanter Akteure gesprochen werden.

Trotzdem unterscheiden sich beide Organisationen im Hinblick auf die Reformentwicklungen in der Folgezeit. Auch was die internen Entscheidungsmodalitäten betrifft, bestehen mit Blick auf das Personalmanagement nur geringfügige Unterschiede, welche die beobachtete Varianz kaum erklären können. Sowohl das OECD-Sekretariat als auch die Kommission sind Teil eines politisch-administrativen Systems, in dem der Rat der Mitgliedsstaaten über die Allokation ihrer finanziellen und personellen Ressourcen 
und somit auch über ihre grundlegenden administrativen Strukturen entscheidet (Rittberger/Zangl 2003).

Neben den genannten Faktoren Problemdruck und Entscheidungsverfahren sind weitere endogene Merkmale denkbar, welche die Reformunterschiede zwischen beiden Organisationen erklären könnten. Hierzu zählen etwa die Größe der Organisationen oder ihr Aufgabenspektrum und, damit verbunden, mögliche Kompetenzunterschiede. Wenngleich diese Aspekte in bestimmten Fällen durchaus von Bedeutung sein mögen, so erscheinen sie von eher nachrangiger Relevanz, wenn es darum geht, Reformunterschiede im Bereich des Personalmanagements zu erklären. So besteht kein direkter $\mathrm{Zu}$ sammenhang zwischen der Größe, den Aufgaben und den Kompetenzen einer Organisation und potenziellem Reformbedarf im Bereich des Personalmanagements (vgl. auch allgemein Knill/Bauer 2007). Dies gilt zumindest dann, wenn die Organisation über eine gewisse Mindestzahl an Mitarbeitern verfügt (also etwa nicht nur aus einem kleinen Sekretariat besteht) und eine gewisse Bandbreite an Aufgaben abdeckt. Beides ist für die hier untersuchten Organisationen anzunehmen, sodass diese Aspekte für die weitere Untersuchung nicht näher betrachtet werden.

Vor diesem Hintergrund richtet sich das Augenmerk unseres Erklärungsansatzes daher insbesondere auf Unterschiede, die sich aus dem Umfeld der beiden Organisationen ergeben könnten. In diesem Zusammenhang bieten sich Theorien des institutionellen Isomorphismus als Rahmen an, um das Phänomen der internationalen Diffusion und Homogenisierung von Politikinnovationen und Reformkonzepten im Hinblick auf Verwaltungsreformen zu erklären (DiMaggio/Powell 1991; Meyer/Rowan 1977; Meyer et al. 1997; Levi-Faur 2002). Grundlegend für diese theoretische Argumentationsrichtung ist, dass Legitimation gegenüber der für die Organisation relevanten Umwelt die wesentliche Grundlage für Wandel darstellt. Weniger die Funktionalität von in der Organisationsumwelt verwendeten Strukturen und Modellen, sondern das Ausmaß ihrer Institutionalisierung in der relevanten Umwelt entscheidet darüber, $\mathrm{ob}$ - wie in unserem Fall - das Personalmanagement einer Organisation seitens der involvierten Akteure als „angemessen“ wahrgenommen wird (March/Olsen 1989; Walgenbach 1998: 272 f.). Dieser Sichtweise zufolge wird der Wandel von Organisationen also eher von externen Entwicklungen als von organisationsinternen Debatten über die Funktionalität und Effizienz vorhandener Strukturen beeinflusst.

Ursache für den Wandel innerhalb einer Organisation ist damit das Handeln anderer Organisationen in ihrem Umfeld. Je mehr sich in diesem Umfeld ein bestimmtes Reformmodell als dominantes, da von einer großen Zahl von Organisationen übernommenes und als erfolgreich erachtetes, Konzept herausstellt, desto größer ist der Anreiz für eine Organisation, dieses Konzept ebenfalls zu übernehmen. Die Theorie des Isomorphismus weist somit enge Bezüge zur politikwissenschaftlichen Diffusions- und Konvergenzforschung auf. Mit der Übernahme eines dominanten Reformmodells verbindet sich die Erwartung einer Angleichung und damit einer Konvergenz von Organisationsstrukturen im Zeitverlauf. Voraussetzung für diese Konvergenz ist die internationale Diffusion von Reformkonzepten (Holzinger et al. 2007). Die hinter diesen Prozessen der Diffusion und Konvergenz stehenden Kausalmechanismen können sehr unterschiedlicher Natur sein und von Zwang über rechtliche Harmonisierung bis hin zu transnationaler Kommunikation und Politiklernen reichen (Holzinger/Knill 2007). 
Diese Faktoren decken sich weitgehend mit den drei Mechanismen, die DiMaggio und Powell (1991) identifizieren, um solche extern stimulierten Anpassungsprozesse zu erklären: erzwungener, mimetischer und normativer Isomorphismus.

Im Folgenden wird die Relevanz dieser Faktoren zur Analyse der Reformunterschiede zwischen OECD-Sekretariat und EU-Kommission näher betrachtet. Dabei zeigt sich, dass auch diese Aspekte keine hinreichende Basis für eine Erklärung bieten. Im Gegenteil: Es wäre ein genau umgekehrtes Szenario zu erwarten gewesen, dem zufolge Entwicklungen in Richtung NPM im OECD-Sekretariat weitaus ausgeprägter hätten sein müssen als in der Kommission. Ein besseres Verständnis der empirischen Befunde wird erst möglich, wenn - so die Argumentation am Ende dieses Abschnitts - die Theorie isomorphen Wandels ergänzt bzw. partiell modifiziert wird.

\subsection{Erzwungene Isomorphie}

Im Falle von erzwungener Isomorphie passen Organisationen ihre Strukturen und Prozeduren an solche Organisationen an, von denen sie finanziell oder rechtlich abhängig sind. DiMaggio und Powell (1991: 74) stellen die Hypothese auf, dass „the greater the dependence of an organization on another organization, the more similar it will become to that organization in structure, climate, and behavioural focus".

In Bezug auf die Reform des Personalmanagements einer internationalen Organisation, könnte diese Abhängigkeit aus dem potenziellen Druckpotenzial der Mitgliedsstaaten resultieren. In beiden hier untersuchen Organisationen spielen die Mitgliedsstaaten die entscheidende Rolle bei der Allokation der finanziellen und personellen Ressourcen. Es wäre demnach denkbar, dass Mitgliedsstaaten dahingehend Druck ausüben, dass die bestehenden Managementstrukturen entsprechend reformiert werden. Wir gehen davon aus, dass die Abhängigkeit einer internationalen Organisation von ihren Mitgliedsstaaten durch drei Faktoren bestimmt wird.

Erstens variieren die Finanzbeiträge der Mitgliedsstaaten, wodurch OECD-Sekretariat und Europäische Kommission de facto in unterschiedlicher Art und Weise von Mitgliedsstaaten abhängig sind. Im Hinblick auf unsere Forschungsfrage ist es von besonderer Bedeutung, ob eine internationale Organisation eher von Mitgliedsstaaten abhängig ist, die selbst weitreichende Reformen durchgeführt haben (NPM-Kernanwender), oder eher von Staaten, die als NPM-Nachzügler gelten. Um diese Varianz zu messen, teilen wir die Mitgliedsstaaten der OECD und der Europäischen Union (EU) in die genannten zwei Gruppen ein und betrachten den jeweiligen Stimmenanteil und den finanziellen Anteil an den Mitgliedsbeiträgen für beide Gruppen. Die Einteilung der Mitgliedsstaaten basiert dabei auf der üblichen Klassifizierung in der Literatur. ${ }^{4}$

Zweitens ist anzunehmen, dass der Druck der Mitgliedsstaaten auf die Einführung eines als effizient wahrgenommenen Personalmanagements steigt, je höher der Anteil der Personalausgaben am Gesamtbudget der jeweiligen Organisation ist. Umgekehrt ist drittens der (finanzielle) Druck der Mitgliedsstaaten umso geringer einzuschätzen, je

4 Hierbei wird von einer zeitlich konstanten Ausprägung von NPM in den Mitgliedsstaaten ausgegangen. Die zehn neuen Mitgliedsstaaten der EU werden nicht berücksichtigt, da sie über die Reform des Personalmanagements der Europäischen Kommission im Jahre 2004 faktisch nicht mitentscheiden konnten. 
Tabelle 5: NPM-Kernanwender und NPM-Nachzügler in der EU und in der OECD

\begin{tabular}{|l|l|l|}
\hline Gruppe & \multicolumn{1}{|c|}{ EU } & \multicolumn{1}{|c|}{ OECD } \\
\hline NPM-Kernanwender & $\begin{array}{l}\text { Dänemark, Finnland, Großbri- } \\
\text { tannien, Irland, Niederlande, } \\
\text { Schweden }\end{array}$ & $\begin{array}{l}\text { Australien, Dänemark, Finnland, } \\
\text { Großbritannien, Irland, Kanada, } \\
\text { Neuseeland, Niederlande, Norwegen, } \\
\text { Schweden, Schweiz, USA }\end{array}$ \\
\hline NPM-Nachzügler & $\begin{array}{l}\text { Belgien, Deutschland, Frank- } \\
\text { reich, Griechenland, Italien, Ös- } \\
\text { terreich, Portugal, Spanien }\end{array}$ & $\begin{array}{l}\text { Belgien, Deutschland, Frankreich, } \\
\text { Griechenland, Italien, Japan, Mexiko, } \\
\text { Österreich, Portugal, Spanien, Südkorea, } \\
\text { Türkei }\end{array}$ \\
\hline $\begin{array}{l}\text { Sonstige } \\
\text { (keine Angaben zu NPM) }\end{array}$ & Luxemburg & $\begin{array}{l}\text { Island, Luxemburg, Polen, Slowakei, } \\
\text { Tschechische Republik, Ungarn }\end{array}$ \\
\hline
\end{tabular}

Quelle: Eigene Darstellung, basierend auf Hood (1995); OECD (1995); Pollitt/Bouckaert (2004); Kim (2002); Sahlin-Andersson (2002).

mehr die internationale Organisation auch über eigene, von mitgliedsstaatlichen Beiträgen unabhängige Einnahmen verfügt.

Im Rat der OECD hat jedes Land eine Stimme, im Rat der EU werden die Stimmen dagegen gewichtet. Setzt man nun die Stimmenanteile der jeweiligen Gruppen ins Verhältnis zueinander, so kommt man zu folgendem Ergebnis: In der EU verfügen die NPM-Nachzügler über fast doppelt so viele Stimmen wie die NPM-Kernanwender, während in der OECD beide Gruppen in etwa gleich viele Stimmen haben. Eine ähnliche Varianz ergibt sich bei der Betrachtung der Zusammensetzung des Budgets. Während mehr als die Hälfte der Einnahmen im EU-Budget aus NPM-Nachzüglern wie Deutschland, Frankreich, Italien oder Spanien stammen, bezieht die OECD immerhin ein Drittel ihrer Einnahmen von NPM-Kernanwendern wie den USA, Großbritannien und Kanada. Die Kommission verfügt zudem über einen bedeutenden Anteil eigener Einnahmen, der ihr, im Gegensatz zur OECD, eine gewisse finanzielle Autonomie verleiht. Auch ist der Anteil der Personalausgaben am Gesamthaushalt für die EU mit 2,5 Prozent sehr gering - insbesondere im Vergleich zu den Ausgaben im Bereich Agrarund Strukturpolitik (vgl. Europäische Kommission 2006a). In der OECD hingegen machen die Personalausgaben fast die Hälfte des Budgets aus, jeweils etwa 20 Prozent sind für Pensions- bzw. Betriebskosten reserviert. Reformen im Bereich Personal dürften daher in der OECD von besonderer Bedeutung sein, da hier grundsätzlich hohe Einsparmöglichkeiten bestehen. Die folgende Tabelle fasst die Charakteristika der OECD und der Kommission in Bezug auf die drei genannten Indikatoren zusammen.

Insgesamt wird deutlich, dass für das OECD-Sekretariat eine gegenüber der Kommission deutlich stärkere Annäherung an NPM-Prinzipien zu erwarten gewesen wäre; unsere empirischen Ergebnisse zeigen aber genau das Gegenteil. Erzwungene Isomorphie vermag somit unsere empirischen Befunde nicht zufriedenstellend zu erklären. 
Tabelle 6: Indikatoren von Reformen durch erzwungene Isomorphie, in Prozent

\begin{tabular}{|l|c|c|}
\hline Indikatoren & KOM & OECD \\
\hline Stimmenanzahl im Rat & 32,1 & 36,7 \\
$\ldots$ von NPM-Kernanwendern & 65,6 & 43,3 \\
... von NPM-Nachzüglern & 2,3 & 20,0 \\
... von anderen Mitgliedsstaaten & & \\
\hline Haushaltseinnahmen und -ausgaben & 27,0 & 7,3 \\
Anteil eigener Einnahmen & 19,6 & $33,1^{*}$ \\
$\ldots$ von NPM-Kernanwendern & 53,2 & $41,2^{*}$ \\
$\ldots$ von NPM-Nachzüglern & 0,2 & 1,0 \\
... von anderen Mitgliedsstaaten & 2,5 & 47,2 \\
\hline Anteil der Personalkosten am Budget & &
\end{tabular}

* = plus einem öffentlich nicht zugänglichen Anteil freiwilliger Beiträge dieser Mitgliedstaaten

Quelle: Eigene Darstellung, basierend auf Europäische Kommission (2006a); OECD (2006a, 2006b).

\subsection{Mimetische Isomorphie}

Die Anpassung von Organisationsstrukturen an Modelle, die im Umfeld der Organisation verwendet werden, muss nicht notwendig über Zwang vermittelt werden, sondern kann auch im Sinne einer freiwilligen Übernahme in einem Zustand hoher Unsicherheit erfolgen. In einer solchen Situation, so die theoretische Vermutung, besteht eine hohe Wahrscheinlichkeit dafür, dass Innovationen, die im Organisationsumfeld als erfolgreich gelten, imitiert oder kopiert werden. Anstatt aufwändig nach eigenen Lösungen für bestehende Probleme zu suchen, sind Organisationen bestrebt, ihre Legitimation durch die Imitation dominanter Regeln zu sichern (DiMaggio/Powell 1991: 75; Guler et al. 2002: 213). Unsicherheit kann sich dabei aus mehreren Ursachen ergeben. Sie kann einerseits kognitiver Natur sein, etwa wenn Organisationsziele und ZweckMittel-Relationen unklar sind oder die Organisation mit neuartigen Problemen konfrontiert wird. Unsicherheit kann aber auch aus fehlender Planungssicherheit im Hinblick auf finanzielle oder personelle Ressourcen der Organisation erwachsen.

Um solche Effekte potenzieller Unsicherheit zu messen, die möglicherweise die Reform des Personalmanagements in der Europäischen Kommission und der OECD beeinflusst haben, konzentrieren wir uns aus Gründen der empirischen Messbarkeit im Folgenden auf den Aspekt der Ressourcenplanung. Inwiefern können sich beide Organisationen der finanziellen und personellen Mittel zur Erfüllung ihrer Aufgaben sicher sein? Als Indikatoren bieten sich die zeitliche Gültigkeit des Haushalts sowie die Entwicklung der Einnahmen und der Beschäftigtenzahlen im Untersuchungszeitraum an. ${ }^{5}$ Je unsicherer die Situation einer Organisation in Bezug auf diese Aspekte ist (ausgedrückt durch eine kurze Gültigkeit des Haushalts und sinkender Einnahmen und Beschäftigtenzahlen), desto mehr sollte die Organisation Reformen im Sinne des dominanten Leitbilds NPM nachahmen.

5 Die Wahl dieser Indikatoren hebt auf die lang- bzw. mittelfristige Planungssicherheit der Organisationen ab. Es geht nicht darum, inwieweit die Mitgliedsstaaten über budgetäre Druckausübung entsprechende Reformprozesse durchsetzen möchten - ein Aspekt, der im Bereich der erzwungenen Isomorphie einzuordnen wäre. 
Betrachtet man die empirischen Ausprägungen dieser Indikatoren, so zeigt sich erneut, dass fundamentale Reformen in Richtung NPM eher im OECD-Sekretariat als in der Kommission zu erwarten gewesen wären. Dies wird zunächst daran deutlich, dass die Kommission über eine höhere Planungssicherheit bei der Haushaltsführung verfügt. In der EU entscheiden die Mitgliedsstaaten alle sieben Jahre über den Finanzrahmen des Haushalts, in der OECD geschah dies bis 2006 jährlich, ab 2007 nun in zweijährigem Rhythmus (Interview, 17.03.2006). Hierbei muss der Rat sowohl den Haushalt als auch das Arbeitsprogramm des Generalsekretärs einstimmig beschließen.

Das OECD-Sekretariat war also ständig möglichem finanziellem Druck der Mitgliedsstaaten ausgesetzt. Umgekehrt bewirkt die auf sieben Jahre ausgerichtete Haushaltsplanung der EU, dass die Unsicherheit der Kommission bezüglich ihrer finanziellen und personellen Ressourcen eher gering einzuschätzen ist. Im Finanzrahmen der EU werden der Kommission konstante Einnahmen und eine stabile Zahl von Planstellen zugesichert. In der OECD kam es dagegen Ende der 1990er Jahre zu einer massiven finanziellen „Einnahmekrise“. Die Mitgliedsstaaten kürzten das Budget des Sekretariats zwischen 1995 und 1999 um real 18 Prozent, woraufhin 220 Stellen gestrichen wurden (OECD 1997a: 93, 1999: 32). Auslöser dieser Budgetkrise waren die USA, die aufgrund interner Dispute bezüglich der Beitragszahlungen an internationale Organisationen ihren Mitgliedsbeitrag für die OECD komplett einfroren (Chavranski 1997: 72 f.). Nach einigen Monaten wurden diese Zahlungen zwar wieder aufgenommen, dabei jedoch dauerhaft um 10 Prozent gesenkt. Als Reaktion senkten auch die anderen Mitgliedsstaaten ihre absoluten Mitgliedsbeiträge. Die Gehälter der OECD-Mitarbeiter mussten zu dieser Zeit gekürzt und mit Verspätung ausgezahlt werden. Erst im Jahr 2000 konnten die Einnahmen wieder stabilisiert werden; sie wachsen jedoch seitdem nur sehr geringfügig. Die Anzahl der Mitarbeiter ist heute um 10 Prozent niedriger als 1995, obwohl in der Zwischenzeit fünf neue Mitgliedsstaaten (Polen, Slowakei, Südkorea, Tschechische Republik, Ungarn) der OECD beitraten. Nominal lag der OECDHaushalt im Jahr 2005 unter seinem Ausgangswert von 1995 (OECD 2005b). Ein Mitarbeiter drückte die daraus folgenden Konsequenzen wie folgt aus: „You get to have to do the same amount of work with much less money" (Interview, 17.03.2006). Im Vergleich zur komfortablen Situation in der Europäischen Kommission kann daher die Planungssicherheit des OECD-Sekretariats hinsichtlich seiner personellen und finanziellen Ressourcen als prekär bezeichnet werden. Diese Varianz führte aber offensichtlich nicht dazu, dass das Personalmanagement des OECD-Sekretariats auch entsprechend weitreichender an Prinzipien des NPM angepasst wurde.

\subsection{Normative Isomorphie}

Ein weiterer Mechanismus, der freiwilligen isomorphen Wandel auslösen kann, basiert auf ähnlichen Denkhaltungen und professionellen Orientierungen der Mitarbeiter verschiedener Organisationen. In diesem Kontext wird vor allem der Einfluss ähnlicher Bildungshintergründe und die Rolle von epistemischen Gemeinschaften (Haas 1992) betont, die für das gemeinsame Verständnis von Policy-Problemen und Lösungen verantwortlich sind (Hasse/Krücken 2005: 26). 
Beide hier untersuchten Organisationen weisen grundsätzlich relativ ähnliche Strukturen im Hinblick auf die professionelle Orientierung und Ausbildung insbesondere ihres akademischen Personals auf. Der akademische Mitarbeiterstab beider Organisationen zählt zu dem am besten ausgebildeten in Europa (Hooghe 2001; Spescha 2005). Es erscheint daher zweckmäßiger, nicht die Ausbildung, sondern das Herkunftsland der Mitarbeiter als prägendes Element ihrer Denkhaltung heranzuziehen. So stellt etwa Hooghe (2001) in ihrer empirischen Studie über die Hintergründe von Entscheidungen in der Europäischen Kommission heraus, dass deren Beamte ihre Entscheidungen zumeist an der „Mehrheitsmeinung“ ihrer Heimatländer orientieren. Überträgt man diese empirische Erkenntnis auf organisationsinterne Diskussionen über die konkrete Gestaltung von Personalmanagement, so ist zu vermuten, dass Mitarbeiter diejenige Ausgestaltung favorisieren, die sie auch aus ihren Heimatländern als dominantes Konzept kennen. Demnach wäre in einer internationalen Organisation zu erwarten, dass angelsächsische Mitarbeiter eine Reform des Personalmanagements (d. h. eine Anpassung an NPM) stärker propagieren als Deutsche oder Franzosen.

Zwei Faktoren relativieren allerdings eine mögliche normative Bindung der Mitarbeiter: die Dauer ihrer Zugehörigkeit zur Organisation und das Ausmaß ihrer diskursiven Beschäftigung mit Fragen des Personalmanagements. Je länger ein Mitarbeiter einer bestimmten internationalen Organisation angehört, desto stärker wird er nun durch die Organisation und nicht mehr durch Denkhaltungen des Heimatlandes sozialisiert (Hooghe 2001: 211). Im Hinblick auf das Ausmaß der diskursiven Beschäftigung der Mitarbeiter mit Personalmanagement ist zu erwarten, dass sie innerhalb einer internationalen Organisation umso mehr zu „Diffusionsagenten“ (Hasse/Krücken 2005: 24) von NPM werden, je stärker sie sich mit anderen Akteuren ihrer relevanten Umwelt diskursiv über Personalmanagement austauschen. In diesem Zusammenhang ist von entscheidender Bedeutung, ob und inwieweit sich die jeweiligen Organisationen selbst federführend mit dem Thema Personalmanagement befassen.

Um die Kommission und das OECD-Sekretariat anhand dieser Indikatoren zu untersuchen, konzentrieren wir uns auf das akademisch ausgebildete Personal und die Führungskräfte in beiden Organisationen. Hierzu zählen die OECD-Mitarbeiter der Laufbahn A (2006: 906 Mitarbeiter) und in der Kommission die Mitarbeiter der gleichnamigen ehemaligen Laufbahn (2002: 7482 Mitarbeiter). ${ }^{6} \mathrm{Zu}$ den Führungskräften des OECD-Sekretariats (Besoldungsgruppen A6-A7) zählen 53 Mitarbeiter, zu denen der Europäischen Kommission (Besoldungsgruppen A1, A2) 265 Mitarbeiter.

In der Kommission stammen sowohl das Personal insgesamt als auch die Führungskräfte zu mehr als zwei Dritteln aus Ländern, die als NPM-Nachzügler gelten (insbesondere Deutschland, Frankreich, Belgien, Italien und Spanien). Weniger als 30 Prozent der Führungskräfte kommen aus Vorreiterländern, was die Einführung von NPM betrifft. Zur letztgenannten Gruppe zählen vor allem die Briten (10,7 Prozent). Im OECD-Sekretariat kommen indessen die meisten Führungskräfte aus den NPM-Vorreiter-Staaten (insbesondere aus den USA und Großbritannien), während sich bezogen auf das gesamte Personal Mitarbeiter aus NPM-Nachzüglern und NPM-Kernanwen-

6 Für beide Organisationen können die aktuellen Daten verwendet werden, da sich die Zusammensetzung des Personals bezüglich der Nationalitäten in den letzten zehn Jahren nur marginal verändert hat. 
Tabelle 7: Nationalität der Mitarbeiter und Führungskräfte in der Europäischen Kommission und im OECD-Sekretariat, in Prozent

\begin{tabular}{|l|r|r|}
\hline Indikatoren & KOM & OECD \\
\hline Akademisch ausgebildetes Personal & 25,3 & 48,5 \\
$\ldots$ aus NPM-Kernanwendern & 73,9 & 49,0 \\
$\ldots$ aus NPM-Nachzüglern & 0,8 & 2,5 \\
$\ldots$ aus anderen Mitgliedsstaaten & & 56,6 \\
\hline Führungskräfte & 29,8 & 43,4 \\
$\ldots$ aus NPM-Kernanwendern & 68,7 & 0,0 \\
$\ldots$ aus NPM-Nachzüglern & 1,5 & \\
\hline aus anderen Mitgliedsstaaten & & \\
\hline
\end{tabular}

Quelle: Eigene Darstellung, basierend auf Europäische Kommission (2006b); OECD (2004).

dern in etwa die Waage halten. Der Vergleich zwischen beiden Organisationen - bezogen auf die Nationalität ihres Personals und ihrer Führungskräfte - ist in Tabelle 7 zusammengefasst.

Die Angaben machen deutlich, dass der normative Druck, das Personalmanagement zu reformieren, im OECD-Sekretariat höher einzuschätzen ist als in der Kommission. Diese Aussage wird auch durch die Betrachtung der Vertragsdauer der Mitarbeiter gestützt: Im Sekretariat der OECD verfügen nur 30 Prozent der Führungskräfte und 45 Prozent der restlichen Mitarbeiter über unbefristete Verträge; in der Kommission haben hingegen fast alle Mitarbeiter Verträge auf Lebenszeit. Dadurch dürfte im OECD-Sekretariat tendenziell ein größerer Austausch von Personal und damit von neuen Ideen stattfinden als in der Europäischen Kommission. ${ }^{7}$

Auch wenn man die Einbindung von Mitarbeitern in internationale Gruppen und Netzwerke betrachtet, in denen Reformen des Personalmanagements diskutiert werden, wäre es wahrscheinlicher, dass größere Reformanstrengungen in Richtung NPM eher im OECD-Sekretariat als in der Kommission betrieben werden. Während sich die Kommission in erster Linie mit der Entwicklung und Formulierung von Politiken in den verschiedenen, von der Gemeinschaft abgedeckten Politikbereichen beschäftigt, betrachtet die OECD sich selbst als "think tank“ (Interview, 15.03.2006). Sie verfügt über 200 Komitees und Arbeitsgruppen, in denen sich jährlich annähernd 40000 hohe Fachbeamte aus den Mitgliedstaaten mit den Mitarbeitern der OECD über „best practice-Modelle" austauschen (Baldi/Beglinger 2005). Die Politikdiskussion in den Komitees der OECD verfolgt dabei vor allem das Ziel, Lernprozesse zu stimulieren, gemeinsame Lösungsansätze zu entwickeln und diese im Rahmen des „peer-review-Verfahrens" zu evaluieren (Schlögl 2005: 5). Mittels ihres PUMA-Komitees agierte die OECD als der internationale Fürsprecher von New Public Management (OECD 1995; Sahlin-Andersson 2002).

Offenkundig lässt sich daher aus den bislang betrachteten Mechanismen der Isomorphie keine zufriedenstellende Erklärung für Unterschiede in den Reformen des Personalmanagements ableiten. Dies impliziert, bezogen auf die OECD, eine paradoxe

7 Es gibt innerhalb der OECD auch einen festen Mitarbeiterstamm: Die Hälfte aller aktuellen Mitarbeiter der Laufbahn A arbeitet seit über fünf Jahren in der OECD, insgesamt 25 Prozent von ihnen sogar seit mehr als 15 Jahren (OECD 2004). 
Konstellation: Diejenige Organisation, die NPM nach außen mit am stärksten propagierte, erweist sich als Nachzügler, wenn es darum geht, diese Reformelemente bei sich selbst entsprechend umzusetzen. Warum dies so ist, soll im folgenden Abschnitt geklärt werden. Es wird argumentiert, dass diese Frage durch eine nähere Betrachtung der Wirkungsbedingungen der Mechanismen isomorphen Wandels beantwortet werden könnte.

\subsection{Bedingungen und Grenzen isomorphen Wandels}

Die Theorie des institutionellen Isomorphismus ermöglicht es, zwischen verschiedenen Mechanismen des Wandels zu unterscheiden. Außerdem identifiziert die Theorie Faktoren, welche die relative Bedeutung jedes Mechanismus in einer bestimmten Konstellation bestimmen; z. B. ist die erzwungene Isomorphie umso stärker ausgeprägt, je mehr die Organisation von ihrem Umfeld abhängig ist. Theoretisch nicht hinreichend berücksichtigt ist jedoch bislang, dass Organisationen, die nach Legitimation in ihrer relevanten Umwelt streben, in vielen Fällen mit einer komplexen und ambivalenten Auswahlsituation konfrontiert sind. Auf der einen Seite könnte mehr als ein dominantes Modell als Blaupause für eine Reform herangezogen werden. Auf der anderen Seite, und das ist von besonderer Bedeutung in unserem Fall, kann sich isomorpher Wandel auf verschiedene Dimensionen, Ebenen oder Aspekte bestehender Organisationsstrukturen, Routinen und Praktiken beziehen. Mit anderen Worten: Es ist unklar, unter welchen Bedingungen sich eine Organisation für welche Reform entscheidet, ob sie also etwa ihre Rechnungslegung, ihre Entscheidungsstrukturen oder ihr Personalmanagement reformiert. Welche Elemente des Menüs von potenziell legitimationsfördernden Reformmodellen, die in der internationalen Umwelt zirkulieren, wählt die Organisation aus und warum?

Unsere Befunde legen die Vermutung nahe, dass Organisationen diese Entscheidungen von den relativen Legitimationsgewinnen der jeweiligen Optionen abhängig machen. Die Höhe dieser Gewinne wiederum wird primär durch organisationsinterne Faktoren beeinflusst. Insofern basiert die hier vorgestellte theoretische Argumentation auf einer Verbindung exogener und endogener Faktoren von Organisationswandel. Konkret folgt hieraus, dass die Frage, welcher Legitimationsgewinn mit der Übernahme bestimmter Modelle aus dem Organisationsumfeld einhergeht, von der jeweiligen Problemkonstellation beeinflusst wird, mit der eine Organisation konfrontiert ist. Diese Konstellation wird durch eine Reihe von endogenen Faktoren bestimmt, wie etwa dem Ausmaß und der Art intern wahrgenommener Probleme sowie internen Entscheidungsstrukturen und -verfahren.

Man könnte nun einwenden, dass exakt jene Faktoren am Anfang dieses Kapitels zunächst als potenzielle Erklärungen ausgeschlossen wurden, da diesbezüglich keine Varianz zwischen OECD-Sekretariat und Kommission festzustellen ist. Diese Aussage gilt allerdings nur insofern, als der Ausgangspunkt ein bestimmtes Modell (in unserem Fall NPM im Bereich Personalmanagement) ist und danach gefragt wird, wie die organisationsinterne Konstellation im Hinblick auf die konkreten Anknüpfungspunkte dieses Modells beschaffen ist. Diese Perspektive, die vom konkreten Modell ausgeht, greift jedoch zu kurz, wenn man verstehen will, warum Organisationen bestimmte Innovatio- 
nen übernehmen, andere jedoch nicht. Vielmehr bedarf es hierzu einer breiteren Sichtweise, welche nicht nur einzelne Probleme einer Organisation betrachtet, sondern die jeweilige Gesamtkonstellation der Herausforderungen, mit denen eine Organisation konfrontiert ist. Erst eine solche Betrachtungsweise macht verständlich, warum Organisationen nicht automatisch Politikmodelle übernehmen, die in ihrem Umfeld zirkulieren, sondern diese Übernahmeentscheidung von relativen Legitimationsgewinnen abhängig machen, die mit einem bestimmten Modell in Anbetracht der jeweiligen Problemkonstellation verbunden sind.

Im Falle der OECD wird deutlich, dass diese potenziellen Gewinne in hohem Maße von den spezifischen Legitimationsproblemen einer Organisation beeinflusst werden. Vor dem Hintergrund der fundamentalen Krise, mit der sich die OECD - bezogen auf ihre Identität und ihre zukünftige Stellung im Konzert der internationalen Organisationen - seit Jahren konfrontiert sieht, waren isomorphe Anpassungsprozesse des Personalmanagements kein ausreichendes Mittel zur Überwindung der problematischen Lage. Als Donald Johnston 1996 sein Amt als Generalsekretär der OECD antrat, umschrieb er die Ziele der OECD mit folgenden Worten:

"Since my arrival as Secretary-General on June 1" 1 , 1996, it has become clear to me that the OECD suffers from a lack of a distinct identity, with the breath of its mission poorly understood. Dealing with the full range of public policy issues, it has become increasingly difficult to capture in a few descriptive words what the Organisation actually does" (OECD 1997b: 3).

Zehn Jahre später hält die Suche nach den Zielen der OECD weiter an (Interview, 17.03.2006). ${ }^{8}$ Bevor der neue Generalsekretär Angel Gurría sein Amt im Juni 2006 antrat, bat er die Mitgliedsstaaten eindringlich darum, der OECD ein neues und klares Mandat zu geben, das ihr in Zukunft mehr internationale Relevanz verleihen würde. Die Zielkonflikte und die Identitätskrise der OECD hängen insbesondere mit ihrem auf Einstimmigkeit basierenden Entscheidungsverfahren zusammen. Wenn neue Ziele bestimmt werden, setzt dies grundsätzlich voraus, dass gleichzeitig an anderer Stelle ein Abbau von Aufgaben erfolgt, um den Rahmen des Budgets nicht zu sprengen. Der Rat kann sich jedoch selten einstimmig auf die Beendigung eines Programms oder die Abschaffung einer Arbeitsgruppe einigen (Chavranski 1997: 71; Interview, 17.03.2006). So gibt es immer ein Komitee oder ein Programm, das ein bestimmter Mitgliedsstaat nicht abschaffen will, weil er davon besonders profitiert (Chavranski 1997: 43). Dass nur eine Abkehr von der strikten Konsensentscheidung dieses Problem lösen kann, ist allen Mitgliedsstaaten klar. Doch wie dieses Entscheidungsverfahren aussehen soll, wurde jahrelang ohne Erfolg diskutiert (Interview, 14.03.2006).

Weitere Elemente dieser Zielkonflikte in der OECD sind die Frage ihrer Erweiterung und die Positionierung der OECD gegenüber anderen internationalen Organisationen. Die Debatte, ob die OECD eher die „großen“ Länder Brasilien, Russland, China und Indien aufnehmen soll oder eher neue EU-Staaten wie Litauen, Lettland und Slowenien, hält seit Jahren an (Interview, 17.03.2006). Mittlerweile läuft die OECD außerdem Gefahr, dass ihr andere Organisationen wie der IWF, die Weltbank, die

8 Siehe auch verschiedene Beiträge hierzu in Financial Times 02.03.2003, 30.11.2005; International Herald Tribune 29.11.2005, 10.02.2006; Süddeutsche Zeitung 15.03.2003. 
WTO und die EU im Bereich der Wirtschafts- und Handelspolitik den „Rang ablaufen".

Zusammenfassend ist eine seit mehr als zehn Jahren andauernde Identitätskrise der OECD zu konstatieren, die sich gleichzeitig in einer Legitimationskrise des OECD-Sekretariats gegenüber seiner relevanten Umwelt ausdrückt. Denn anders als etwa bei internationalen Entwicklungsbanken oder Hilfsorganisationen bestimmen die Mitgliedsstaaten der OECD nicht nur Art und Umfang des Arbeitsprogramms, sondern sind in Gestalt ihrer hohen Fachbeamten zugleich wichtigster Adressat dieser Aktivitäten. In Anbetracht einer solchen Situation stellten Reformen des Personalmanagements in Anlehnung an das Leitbild NPM keine signifikanten Legitimationsgewinne bzw. das adäquate Mittel dar, dieser fundamentalen Krise zu begegnen. Die Mitgliedsstaaten verständigten sich stattdessen auf Einsparungen im OECD-Haushalt, die in erster Linie über den Abbau von Personal und drastische Kürzungen des Weiterbildungsbudgets realisiert wurden. Ähnlich fundamentale Zielkonflikte und Herausforderungen ließen sich hingegen für die Europäische Kommission im Untersuchungszeitraum nicht identifizieren. Schon aufgrund ihres siebenjährigen Finanzrahmens und der ihr übertragenen Souveränitätsrechte ist anzunehmen, dass solche Probleme in der Kommission weniger virulent sind als in der OECD.

Diese Betrachtungen legen insgesamt die Vermutung nahe, dass Organisationen sehr selektiv auf die Mechanismen isomorphen Wandels reagieren. Die Effektivität der entsprechenden Mechanismen hängt demnach von den spezifischen Problemen ab, mit denen sich eine Organisation konfrontiert sieht, sowie von der Problemlösungsfähigkeit, die einem bestimmten Modell zugeschrieben wird. Insofern knüpft unsere Argumentation an kontingenztheoretische Überlegungen an, wie sie etwa von March und Olsen (1989) entwickelt wurden.

\section{Fazit}

In diesem Artikel haben wir die Reform des Personalmanagements in der Europäischen Kommission und im OECD-Sekretariat im Zeitraum von 1995 bis 2007 untersucht. Die empirischen Befunde zeigen, dass das Personalmanagement der Kommission weitreichender reformiert wurde und nun eher dem NPM entspricht als das des OECDSekretariats. Dieses Ergebnis ist aus zwei Gründen überraschend. Erstens erscheint es paradox, dass gerade die OECD, die auf internationaler Ebene als zentraler Verfechter von NPM gilt, bei der eigenen Umsetzung dieser Reformen hinterherhinkt. Zweitens steht das Ergebnis im Widerspruch zu den theoretischen Erwartungen, die sich aus dem Konzept des institutionellen Isomorphismus ergeben.

Um vor diesem Hintergrund dennoch zu einer befriedigenden Erklärung der überraschenden Ergebnisse zu gelangen, ist es erforderlich, die Theorie durch Argumente zu ergänzen, welche die spezifischen Bedingungen für die Effektivität der Mechanismen isomorphen Wandels beeinflussen. Unsere Analyse verweist in diesem Zusammenhang darauf, dass die Frage, ob Organisationen international diffundierende Innovationen übernehmen, davon abhängt, inwieweit die Übernahme eines bestimmten Modells potenzielle Legitimationsgewinne verspricht. Letztere sind wiederum von organisationsinternen Faktoren, insbesondere der spezifischen Problemkonstellation, abhängig. 
Anhang

\begin{tabular}{|l|l|l|}
\hline Interviews & \multicolumn{1}{|c|}{ Ort } & \multicolumn{1}{c|}{ Befragte } \\
\hline Interview, 14.03.2006 & Paris (Experteninterview) & - Botschafter eines OECD-Mitgliedsstaates \\
\hline Interview, 15.03.2006 & Paris (Experteninterviews) & - zwei Mitarbeiter der OECD-Personalabteilung \\
\hline Interview, 17.03.2006 & Paris (Experteninterviews) & $\begin{array}{l}\text { - zwei Mitarbeiter der OECD-Personalvertretung } \\
- \text { Botschafter eines OECD-Mitgliedsstaates } \\
- \text { nationaler Sachbearbeiter für } \\
\text { OECD-Personalfragen }\end{array}$ \\
\hline Interview, 12.05.2006 & Brüssel (Experteninterviews) & $\begin{array}{l}- \text { Abgeordneter Sachverständiger für die Reform } \\
\text { des Personalstatuts } \\
- \text { Stabstelle der GD Personal und Verwaltung } \\
\end{array}$ \\
& & $\begin{array}{l}\text { - Beamter der Abteilung für Human Resource } \\
\text { Development }\end{array}$ \\
\hline Interview, 17.05.2006 & Telefoninterviews & $\begin{array}{l}\text { - Botschafter eines OECD-Mitgliedsstaates } \\
- \text { nationaler Sachbearbeiter für } \\
\text { OECD-Personalfragen }\end{array}$ \\
\hline
\end{tabular}

Anzahl nicht zitierter Interviews: 3

\section{Literatur}

Baldi, Marino/Beglinger, Lukas, 2005: Die Schweiz und die Organisation für wirtschaftliche Zusammenarbeit und Entwicklung (OECD), in: Senti, Richard/Ziegler, Andreas R. (Hrsg.), Die Schweiz und die internationalen Wirtschaftsorganisationen. Zürich, 27-46.

Barnett, Michael N./Finnemore, Martha, 1999: The Politics, Power and Pathologies of International Organizations, in: International Organization 53 (4), 699-732.

Barnett, Michael N./Finnemore, Martha, 2004: Rules for the World. International Organizations in Global Politics. Ithaca.

Bauer, Michael W., 2006: Die Reform der Europäischen Kommission: Eine Studie zur Managementmodernisierung internationaler Organisationen, in: Verwaltungsarchiv 97 (3), 270-292.

Bauer, Michael W., 2008: Effects of the Reform of the European Commission on Policy Output, in: Journal of European Public Policy 15 (2), im Erscheinen.

Bauer, Michael W./Knill, Christoph (Hrsg.), 2007: Management Reforms in International Organizations. Baden-Baden.

Bogumil, Jörg/Jann, Werner/Nullmeier, Frank, 2006: Perspektiven der politikwissenschaftlichen Verwaltungsforschung, in: Bogumil, Jörg/Jann, Werner/ Nullmeier, Frank (Hrsg.), Politik und Verwaltung. Politische Vierteljahresschrift Sonderheft 37. Wiesbaden, 9-26.

Bouckaert, Geert, 2006: Auf dem Weg zu einer neo-weberianischen Verwaltung. New Public Management im internationalen Vergleich, in: Bogumil, Jörg/Jann, Werner/Nullmeier, Frank (Hrsg.), Politik und Verwaltung. Politische Vierteljahresschrift Sonderheft 37. Wiesbaden, 354-372.

Chavranski, Henri, 1997: L'OCDE. Au cœur des grands débats économiques. Paris.

Cini, Michelle, 2002: Reforming the European Commission: Discourse, Culture and Planned Change, in: Hosli, Madeleine O./van Deemen, Adrian M.A./Widgrén, Mika (Hrsg.), Institutional Challenges in the European Union. London, 1-21.

Coombes, David, 1970: Politics and Bureaucracy in the European Community. A portrait of the Commission of the EEC. London.

Davies, Michael D.V., 2002: The Administration of International Organizations: Top down and Bottom up. Burlington. 
Demmke, Christoph, 2006: Europäisierung der Personalpolitiken in Europa. Die öffentlichen Dienste zwischen Tradition, Modernisierung und Vielfalt, in: Bogumil, Jörg/Jann, Werner/Nullmeier, Frank (Hrsg.), Politik und Verwaltung. Politische Vierteljahresschrift Sonderheft 37. Wiesbaden, 373-396.

Dijkzeul, Dennis, 1997: The Management of Multilateral Organizations. Den Haag.

Dijkzeul, Dennis/Beigbeder, Yves, 2003: Introduction. Rethinking International Organizations, in: Dijkzeul, Dennis/Beigbeder, Yves (Hrsg.), Rethinking International Organizations. Pathology \& Promise. New York, 1-26.

DiMaggio, Paul J./Powell, Walter W., 1991: The Iron Cage Revisited: Institutional Isomorphism and Collective Rationality in Organizational Fields, in: Powell, Walter W./DiMaggio, Paul J. (Hrsg.), The New Institutionalism in Organizational Analysis. Chicago, 63-82.

Egeberg, Morten, 2006: Balancing Autonomy and Accountability: Enduring Tensions in the European Commission's Development, in: Egeberg, Morten (Hrsg.), Multilevel Union Administration. The Transformation of Executive Politics in Europe. Basingstoke, 31-50.

Europäische Kommission, 2004a: Die Reform der Kommission. Eine Verwaltung im Dienste einer halben Milliarde europäischer Bürger, in: http://ec.europa.eu/reform/2002/index_de.htm; 15.03.2007.

Europäische Kommission, 2004b: Statut der Beamten der Beamten der Europäischen Gemeinschaften. Beschäftigungsbedingungen für die sonstigen Bediensteten der Europäischen Gemeinschaften, in: http://ec.europa.eu/civil_service/docs/toc100_de.pdf; 15.03.2007.

Europäische Kommission, 2005: Mitteilung der Kommission an das Europäische Parlament und den Rat. Bericht über die Umsetzung der Kommissionsreform. Das Mandat ist erfüllt - die Reform geht weiter, in: http://eur-lex.europa.eu/LexUriServ/site/de/com/2005/com2005_0668de01. pdf; 15.03.2007.

Europäische Kommission, 2006a: EU's Budget, in: http://www.europa.admin.ch/eu/info_mat/ dossiers/d/eu_budget.pdf und http://europa.eu.int/comm/budget/budget_glance/where_from_ de.htm; 15.03.2007.

Europäische Kommission, 2006b: Staffing Figures. Staff (officials and temporary agents) Broken down by Gender, Nationality, Grade, Place of Employment, DG and Type of Contract, in: http:// ec.europa.eu/dgs/personnel_administration/staff/index_en.htm; 15.03.2007.

Geri, Laurance R., 2001: New Public Management and the Reform of International Organizations, in: International Review of Administrative Sciences 67 (3), 445-460.

Getz, Heinrich/Jüttner Heinrich, 1972: Personal in internationalen Organisationen. Baden-Baden.

Guler, Isin/Guillén, Mauro F./Macpherson, John M., 2002: Global Competition, Institutions and the Diffusion of Organizational Practices. The International Spread of ISO 9000 Quality Certificates, in: Administrative Science Quaterly 47, 207-233.

Haas, Peter M., 1992: Introduction: Epistemic Communities and International Policy Coordination, in: International Organization 46, 1-36.

Hahn, Hugo J./Weber, Albrecht, 1976: Die OECD. Organisation für Wirtschaftliche Zusammenarbeit und Entwicklung. Baden-Baden.

Hasse, Raimund/Krücken, Georg, 2005: Neo-Institutionalismus. Bielefeld.

Henneberger, Fred, 1997: Arbeitsmärkte und Beschäftigung im öffentlichen Dienst. Eine theoretische und empirische Analyse für die Bundesrepublik Deutschland. Bern u. a.

Holzinger, KatharinalJörgens, Helge/Knill, Christoph (Hrsg.), 2007: Transfer, Diffusion und Konvergenz von Politiken. Politische Vierteljahresschrift Sonderheft 38. Wiesbaden.

Holzinger, Katharina/Knill, Christoph, 2007: Ursachen und Bedingungen internationaler Politikkonvergenz, in: Holzinger, KatharinalJörgens, Helge/Knill, Christoph (Hrsg.), Transfer, Diffusion und Konvergenz von Politiken. Politische Vierteljahresschrift Sonderheft 38. Wiesbaden.

Hood, Christopher, 1991: A Public Management for all seasons?, in: Public Administration 69 (1), 3-19.

Hood, Christopher, 1995: The „New Public Management“ in the 1980s: Variations on a Theme, in: Accounting, Organizations and Society 20 (2/3), 93-109.

Hooghe, Liesbet, 2001: The European Commission and the Integration of Europe. Images of Governance. Cambridge. 
Kassim, Hussein, 2004a: A Historic Accomplishment? The Prodi Commission and Administrative Reform, in: Dimitrakopoulos, Dionyssis G. (Hrsg.), The changing European Commission. Manchester, 33-62.

Kassim, Hussein, 2004b: The Kinnock Reforms in Perspective: Why Reforming the Commission is an Heroic, But Thankless, Task, in: Public Policy and Administration 19 (3), 25-41.

Kieser, Alfred, 2002: Max Webers Analyse der Bürokratie, in: Kieser, Alfred (Hrsg.), Organisationstheorien. Stuttgart, 39-64.

Kim, Pan S., 2002: Civil Service Reform in Japan and Korea: Toward Competitiveness and Competency, in: International Review of Administrative Science 68, 389-403.

Knill, Christoph/Bauer, Michael W., 2007: Theorizing Management Reforms in International Organizations, in: Bauer, Michael W./Knill, Christoph (Hrsg.), Management Reforms in International Organizations. Baden-Baden, 191-199.

Lagreid, Per, 2002: Transforming Top Civil Servant Systems, in: Christensen, Tom/Lagreid, Per (Hrsg.), New Public Management. Burlington, 145-171.

Lequesne, Christian, 1996: La Commission européenne entre autonomie et dependence. Revue française de science politique 46 (3), 389-408.

Levi-Faur, David, 2002: The Politics of Liberalization. Privatisation and Regulation for Competition in Europe's and Latin America's Telecoms and Electricity Industries, in: European Journal of Political Research 42 (5), 705-740.

Levy, Roger, 2003: Critical Success Factors in Public Management Reform. The Case of the European Commission, in: International Review of Administrative Sciences 69, 553-566.

Liebel, Hermann J./Oechsler, Walter A., 1992: Personalbeurteilung. Neue Wege zur Bewertung von Leistung, Verhalten und Potential. Wiesbaden.

Liese, Andrea/Weinlich, Silke, 2006: Die Rolle von Verwaltungsstäben internationaler Organisationen. Lücken, Tücken und Konturen eines (neuen) Forschungsfelds, in: Bogumil, Jörg/Jann, Werner/Nullmeier, Frank (Hrsg.), Politik und Verwaltung. Politische Vierteljahresschrift Sonderheft 37. Wiesbaden, 491-524.

Maguire, Maria, 1993: Pay Flexibility in the Public Sector - an Overview, in: OECD (Hrsg.), Pay Flexibility in the Public Sector. Paris, 9-17.

March, James G./Olsen, Johan P., 1989: Rediscovering Institutions. The Organizational Basis of Politics. New York/London.

McGregor Jr., Eugene B./Solano, Paul, 1996: Data Requirements and Availability, in: Bekke, Hans A. G.M./Perry, James L./Toonen, Theo A.J. (Hrsg.), Civil Service Systems in Comparative Perspective. Bloomington/Indianapolis, 42-64.

Meyer, John W./Frank, David J./Hironaka, Ann/Schofer, Evan/Brandon-Tuma, Nancy, 1997: The Structuring of a World Environmental Regime, 1870-1990, in: International Organization 51, 623-651.

Meyer, John W./Rowan, Brian, 1977: Institutionalized Organizations. Formal Structure as Myth and Ceremony, in: American Journal of Sociology 83, 340-363.

Naschold, Frieder/Bogumil, Jörg, 2000: Modernisierung des Staates. New Public Management in deutscher und internationaler Perspektive. Opladen.

OECD, 1995: Governance in Transition. Public Management Reforms in OECD Countries. Paris.

OECD, 1997a: OECD Annual Report 1997. Paris.

OECD, 1997b: The OECD - Challenges and Strategic Objectives: 1997, Note by the Secretary General (Dokument C(97)180).

OECD, 1999: OECD Annual Report 1999. Paris.

OECD, 2001: OECD Annual Report 2001. Paris.

OECD, 2003: OECD Annual Report 2003. Paris.

OECD, 2004: Staff Profile Statistics (Dokument C(2004)48).

$O E C D$, 2005a: Modernising Government. The Way Forward. Paris.

OECD, 2005b: OECD Annual Report 2005. 45th Anniversary. Paris.

$O E C D$, 2006a: Financial Statement of the Organisation for Economic Co-operation and Development, in: http://appli1.oecd.org/olis/2006doc.nsf/linkto/exd-bc(2006)1; 15.03.2007.

OECD, 2006b: OECD Annual Report 2006. Paris. 
OECD, 2007: Human Resource Management at the OECD, in: http://www.oecd.org/document/ 8/0,2340,en_2649_34481_20008648_1_1_1_1,00.html; 15.03.2007.

Peterson, John, 2004: The Prodi Commission: Fresh Start or Free Fall?, in: Dimitrakopoulos, Dionyssis G. (Hrsg.), The Changing European Commission. Manchester, 15-32.

Pollitt, Christopher/Bouckaert, Geert, 2004: Public Management Reform. A Comparative Analysis. New York.

Reichard, Christoph, 2005: Personalmanagement, in: Blanke, Bernhard/Bandemer, Stephan von/Nullmeier, Frank/Wewer, Göttrik (Hrsg.), Handbuch zur Verwaltungsreform. Wiesbaden, 229-235.

Reinalda, Bob/Verbeek, Bertjan, 2004: The Issue of Decision Making within International Organizations, in: Reinalda, Bob/Verbeek, Bertjan (Hrsg.), Decision Making within International Organizations. London, 9-41.

Rittberger, Volker/Zangl, Bernhard, 2003: Internationale Organisationen - Politik und Geschichte. Opladen.

Sablin-Andersson, Kerstin, 2002: National, International and Transnational Constructions of New Public Management, in: Christensen, Tom/Lagreid, Per (Hrsg.), New Public Management. Burlington, 43-72.

Schedler, Kuno/Proeller, Isabella, 2003: New Public Management. Bern.

Schlögl, Herwig, 2005: OECD im Wandel - gestern, heute, morgen, in: Die Volkswirtschaft 78 (4), 4-7.

Spence, David, 1997: Staff and Personnel Policy in the Commission, in: Edwards, Geoffrey/Spence, David (Hrsg.), The European Commission. London, 68-100.

Spescha, Geli, 2005: OECD und ihr Einfluss auf die nationale Politik - ein Streitgespräch, in: Die Volkswirtschaft 78 (4), 29-33.

Stevens, Anne/Stevens, Handley, 2001: Brussels Bureaucrats? The Administration of the European Union. Basingstoke.

Stone, Diane, 2004: Transfer Agents and Global Networks in the „Transnationalization“ of Policy, in: Journal of European Public Policy 11 (3), 545-566.

Vaanholt, Silke, 1997: Human Resource Management in der öffentlichen Verwaltung. Wiesbaden.

Walgenbach, Peter, 1998: Personalpolitik aus der Perspektive des Institutionalistischen Ansatzes, in: Martin, Albert/Nienhüser, Werner (Hrsg.), Personalpolitik. Wissenschaftliche Erklärungen der Personalpraxis. München/Mering, 267-290.

Wille, Anchrit, 2007: Senior Officials in a Reforming European Commission: Transforming the Top?, in: Bauer, Michael W./Knill, Christoph (Hrsg.), Management Reforms in International Organizations. Baden-Baden, 37-50. 